\title{
Casual 3D Photography
}

\author{
PETER HEDMAN, University College London* \\ SUHIB ALSISAN, Facebook \\ RICHARD SZELISKI, Facebook \\ JOHANNES KOPF, Facebook
}

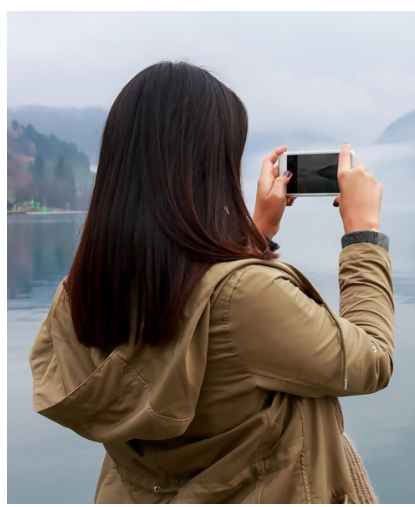

Casual 3D photo capture

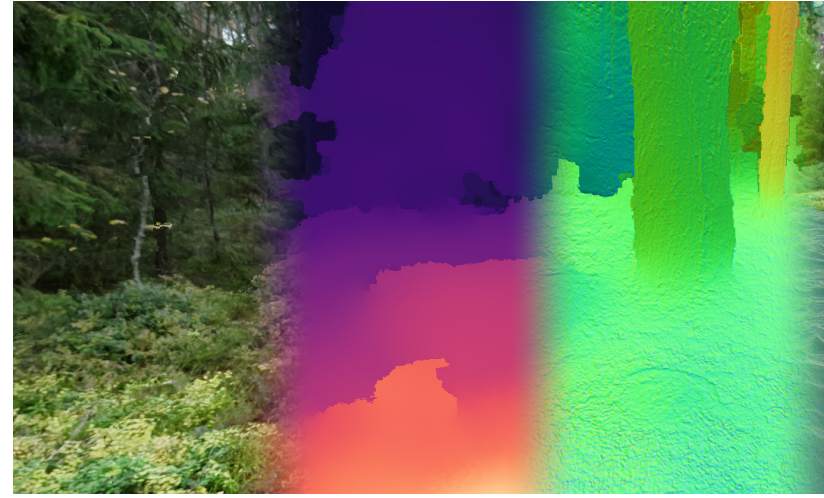

Color
Normal map
Depth Reconstruction

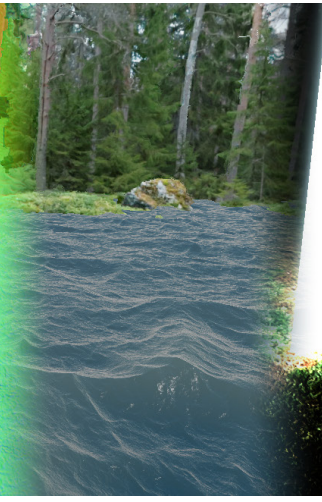

Geometry-aware I Example Effects

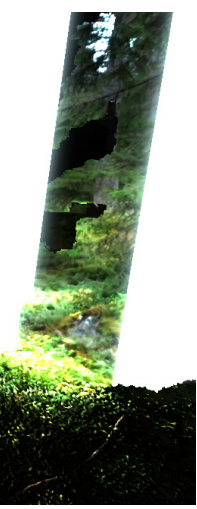

Lighting

Fig. 1. Our algorithm reconstructs a 3D photo, i.e., a multi-layered panoramic mesh with reconstructed surface color, depth, and normals, from casually captured cell phone or DSLR images. It can be viewed with full binocular and motion parallax in VR as well as on a regular mobile device or in a Web browser. The reconstructed depth and normals allow interacting with the scene through geometry-aware and lighting effects.

We present an algorithm that enables casual 3D photography. Given a set of input photos captured with a hand-held cell phone or DSLR camera, our algorithm reconstructs a $3 D$ photo, a central panoramic, textured, normal mapped, multi-layered geometric mesh representation. 3D photos can be stored compactly and are optimized for being rendered from viewpoints that are near the capture viewpoints. They can be rendered using a standard rasterization pipeline to produce perspective views with motion parallax. When viewed in VR, 3D photos provide geometrically consistent views for both eyes. Our geometric representation also allows interacting with the scene using 3D geometry-aware effects, such as adding new objects to the scene and artistic lighting effects.

Our 3D photo reconstruction algorithm starts with a standard structure from motion and multi-view stereo reconstruction of the scene. The dense stereo reconstruction is made robust to the imperfect capture conditions using a novel near envelope cost volume prior that discards erroneous near depth hypotheses. We propose a novel parallax-tolerant stitching algorithm that warps the depth maps into the central panorama and stitches two color-and-depth panoramas for the front and back scene surfaces. The two panoramas are fused into a single non-redundant, well-connected geometric mesh. We provide videos demonstrating users interactively viewing and manipulating our 3D photos.

\footnotetext{
* This work was done while Peter was an intern at Facebook.

Permission to make digital or hard copies of all or part of this work for personal or classroom use is granted without fee provided that copies are not made or distributed for profit or commercial advantage and that copies bear this notice and the full citation on the first page. Copyrights for components of this work owned by others than the author(s) must be honored. Abstracting with credit is permitted. To copy otherwise, or republish, to post on servers or to redistribute to lists, requires prior specific permission and/or a fee. Request permissions from permissions@acm.org.

(c) 2017 Copyright held by the owner/author(s). Publication rights licensed to Association for Computing Machinery.

0730-0301/2017/11-ART234 \$15.00

https://doi.org/10.1145/3130800.3130828
}

CCS Concepts: • Computing methodologies $\rightarrow$ Image-based rendering; Reconstruction; Computational photography;

Additional Key Words and Phrases: 3D Reconstruction, Image-based Rendering, Virtual Reality

\section{ACM Reference Format:}

Peter Hedman, Suhib Alsisan, Richard Szeliski, and Johannes Kopf. 2017. Casual 3D Photography. ACM Trans. Graph. 36, 6, Article 234 (November 2017), 15 pages. https://doi.org/10.1145/3130800.3130828

\section{INTRODUCTION}

Imagine if you could capture any place and digitally preserve it in a way that allows you or your friends to virtually immerse themselves in the scene and re-experience the sensation of being there. Imagine this was nearly as easy as taking a picture today, using a phone or camera you already own. Such technology would be useful for capturing and sharing spaces you visit with your friends, so they feel more connected to you, or preserving your personally treasured places forever as digital memories.

In this paper, we present technology that enables casual $3 D$ photography, moving us closer towards fulfilling this vision. A person captures a scene by moving a hand-held camera sideways at about a half arm's length, while taking a series of still images (Fig. 1, left), possibly with the help of a dedicated capture app. The capture is unstructured, i.e., the motion does not have to be precisely executed, and takes just seconds to a few minutes, depending on the desired amount of coverage. Given this input, our algorithm automatically reconstructs a $3 D$ photo, i.e., a textured, panoramic, multi-layered geometric mesh representation (Fig. 1, middle). 
The most immersive way to enjoy a 3D photo is in VR. Using the reconstructed geometry, we render stereoscopic perspective views that correctly react to tracked user head motion, i.e., provide binocular and motion-parallax depth cues. On non-VR displays such as smart phones, we can still show motion parallax by rendering from viewpoints on a sphere fitted to the estimated input camera locations. Our geometric representation enables interacting with the 3D photo through geometry-aware and artistic lighting effects (Fig. 1, right).

We specifically designed our representation and reconstruction algorithms for as "casual" as possible capture. We do not require the user to capture a scene from all angles, but just an arc around a single viewpoint. As a result, our 3D photos are most suited for an "armchair exploration" scenario. They look best when viewed from within a volume around the viewpoints that were spanned during capture.

An alternative representation often used for VR capture is the omnidirectional stereo representation (ODS) for capturing and rendering stereoscopic panoramic images and videos, which uses separate $360^{\circ}$ panoramas for the left and right eye [Peleg et al. 2001]. These panoramas are captured either by sweeping a regular camera around a ring [Peleg et al. 2001; Richardt et al. 2013] or from a ring of overlapping video cameras [Anderson et al. 2016; Facebook 2016] ODS provides a binocular depth cue by delivering different images to the eyes, but does not provide motion parallax when the user turns or moves their head. The rendered views are not in a linear perspective projection and exhibit distortions such as bent scene lines and incorrect stereo parallax away from the equator. These artifacts are described in more detail in Section 2. Our system provides binocular and motion parallax depth cues, and therefore a better sense of immersion. Another important difference is that any incorrectly reconstructed geometry in a 3D photo will still be rendered consistently for both eyes, while artifacts in an ODS produce inconsistent views that can cause motion sickness.

Multi-view stereo (MVS) [Furukawa and Hernández 2015] algorithms reconstruct a dense and detailed 3D geometric model from a set of images. This mature field in computer vision has seen over 30 years of research, and several high quality and actively maintained software packages implementing state-of-the-art algorithms are available (see Section 6.2). However, applying these algorithms directly in our scenario produces unsatisfactory results, for the following reasons: (1) our casually captured images violate many common assumptions in MVS algorithms leading to geometric artifacts: they are captured with a narrow baseline, and our scenes are often not fully static and contain large textureless areas; (2) the geometry produced by MVS algorithms is not optimized for a specific viewpoint and often lacks completeness and detail. Our approach uses state-of-the-art reconstruction algorithms as core components, but through several technical innovations, we make them work robustly in our scenario. We provide extensive comparisons against several MVS packages in Section 6.2 and the supplementary material.

The reconstruction of a 3D photo starts with estimating input camera poses and a sparse scene model using an off-the-shelf structurefrom-motion algorithm. This is followed by our three major technical innovations.
(1) A novel near-envelope cost volume prior is used to improve the robustness of MVS depth map reconstruction by discarding erroneous nearby depth hypotheses.

(2) A novel parallax-tolerant stitching algorithm warps the depth maps into a central panoramic domain and stitches two panoramas: one for the front and one for the back (occluded) surface in the scene. Each map is reconstructed in the original image domain to achieve good alignment of image and depth edges. These are then rendered with a regular and an inverted z-test to produce front and back surface warps, which are subsequently stitched into corresponding front and back surface panoramas.

(3) A novel two-layer fusion algorithm that merges the front and back surface panoramas into a consistent, two-layered, textured geometric mesh: the 3D photo.

Our 3D photo representation can be rendered on any modern platform using standard graphics engines. The reconstruction quality is sufficient for moderate viewpoint changes, roughly within the volume spanned by the captured images. We have experimented with a variety of playful geometry-aware and lighting effects that make use of the reconstructed scene geometry.

We have applied our algorithm to numerous 3D photos captured with DSLRs and cell phone cameras. Among these are indoor and outdoor as well as man-made and natural scenes. We compare our 3D photos extensively with results obtained with existing state-ofthe-art reconstruction algorithms, and provide quantitative analysis using the Virtual Rephotography evaluation method [Waechter et al. 2017]. In the supplementary material, we provide datasets consisting of input images, results, and intermediate algorithm stage outputs for all of our scenes, as well as the results and rephotography error maps for 16 variants of competing methods.

\section{PREVIOUS WORK}

Our work builds on a long tradition of image-based modeling and rendering, omnidirectional stereo, panoramic image stitching, multiview stereo and free-viewpoint video, photogrammetry, surface normal estimation, and relighting. In this section, we highlight some of the related work in these areas.

Omnidirectional Stereo: Omnidirectional stereo encompasses a class of techniques for stitching together a pair of left-eye and righteye panoramic images [Anderson et al. 2016; Ishiguro et al. 1990; Peleg et al. 2001; Richardt et al. 2013]. The original techniques for generating such images used strips from moving camera images to create the panoramic images, using either mechanical rotation [Ishiguro et al. 1990; Peleg and Ben-Ezra 1999] or hand-held images [Google 2015; Richardt et al. 2013; Zhang and Liu 2015]. More recent systems enable the capture of stereo panoramic videos using multiple cameras arranged in a ring [Anderson et al. 2016; Facebook 2016].

Unfortunately, omnidirectional stereo images have a number of drawbacks [Anderson et al. 2016], particularly when applied to full spherical images. These include:

(1) non-linear perspective: straight lines in the scene are rendered as bent;

(2) unnatural non-rigid motion: the scene appears to swim or bend above and below the equator as the viewer turns their head; 
(3) the lack of realistic motion parallax as the head is turned;

(4) incorrect stereo parallax away from the equator, most noticeable as the distance to the floor appearing "at infinity", resulting in unpleasant vertigo;

(5) the inability to tilt the head sideways and obtain correct binocular parallax;

(6) un-specified behavior at the poles: a simple 180 vertical sweep leaves gaps.

There are some other stitching methods that are not specifically designed for omnistereo but general stereoscopic image pairs. Luo et al. [2012] describe a method for cloning a manually specified selection from one stereoscopic image to another, and adjusting the colors and disparities through iterative gradient-domain blending.

Parallax-tolerant panorama stitching: Some methods warp input images to stitch artifact-free monocular panoramas [Lin et al. 2016; Shum and Szeliski 1998; Zaragoza et al. 2013; Zhang and Liu 2014]. Recent work[Zhang and Liu 2015] demonstrates that this approach also extends to omni-directional stereo. However, this line of work has not yet produced explicit 3D geometry, making them unable to produce head-motion parallax in VR.

Panoramas with Depth: An alternative to generating a left-right pair of panoramic images is to augment a traditional stitched panoramic image with depth information [Im et al. 2016]. However, simply adding a single depth channel to a panorama is not sufficient, because viewpoint changes can lead to issues at depth discontinuities, i.e., reveal long stretched triangles or holes.

Zheng et al. [2007] create a layered depth panorama from multiple overlapping images using a cylinder-sweep multi-view stereo (MVS) algorithm. One problem with Zheng et al's approach is that their layers are not connected, which prevents their algorithm from reproducing sloped surfaces. In addition, our approach of first computing per-image depth maps allows us to use the original images as references to achieve better edge alignment, and our stitching step can hide MVS artifacts.

Thatte et al. [2016] describe a representation rendering for ODS with depth and sketch out capturing and rendering algorithms, but mostly demonstrate their system with synthetic data and a simple "proof-of-concept" captured scene, and leave the question of reliable depth estimation open.

SLAM and depth sensors: Simultaneous Localization and Mapping (SLAM) methods are able to reconstruct hand-held video [Engel et al. 2016; Mur-Artal and Tardós 2016]. The sparse or semi-dense reconstructions created by these methods are useful for rendering augmented reality overlays onto video, but more geometry- or occlusion-aware effects such as the virtual water surface (Figure 1) or virtual viewpoint changes require dense reconstructions. Dense SLAM methods [Izadi et al. 2011] usually rely on depth sensor input. [Hedman et al. 2016] use a combination of depth sensors and multi-view stereo in an image-based rendering system that reproduces view-dependent effects and allows for a freely moving camera. However, their scene representation is expensive to render and this system requires elaborate, indoor-only capture.

Multi-view Stereo and Free-viewpoint Video: Multi-view stereo algorithms produce depth maps or surface meshes by matching multiple overlapping images [Seitz et al. 2006]. Recent examples of such papers include [Fuhrmann et al. 2014; Furukawa and Ponce 2010; Galliani et al. 2015; Im et al. 2016; Jancosek and Pajdla 2011; Langguth et al. 2016; OpenMVS 2016; Schönberger et al. 2016; Vogiatzis et al. 2007]. Multi-view stereo algorithms are used in commercial photogrammetric tools for 3D reconstruction of VR scenes [Realities 2017; Valve 2016]. They are also used to produce 3D proxies that can be used in image-based rendering applications [Buehler et al. 2001; Chaurasia et al. 2013; Debevec et al. 1996]. A related line of work aims to build 3D models from multiple video streams, which enables the interactive viewing of free-viewpoint video (see [Anderson et al. 2016] for some references).

However, applying these algorithms directly in our scenario yields unsatisfactory results:

(1) Our uncontrolled and imperfect captures typically exhibit a combination of geometric deformations, appearance changes, and slight scene motion. Under these conditions MVS algorithms produce a variety of artifacts, such as erroneous depth or "flying" pieces of geometry.

(2) The reconstructions are not designed to be viewed from a single view point. The geometric details can appear blobby and not well aligned with image edges.

Flynn et al. [2016] provide a nice end-to-end system for imagebased reconstruction and rendering using deep learning. However, the rendering speed is slow, on the order of minutes per displayed frame.

(3) Most algorithms rely on confidence measures that relate a scene point's depth to the baseline of its observing cameras. As a result, many algorithms do not reconstruct far away geometry, where the confidence goes to zero.

We provide an extensive comparative evaluation with several stateof-the-art MVS systems in Sec. 6.2.

Single-Image CNN Depth: Recent work attempts to estimate a depth map from a single image using deep learning techniques [Eigen et al. 2014; Godard et al. 2017; Ummenhofer et al. 2017]. This is a very promising line of research, but the results have not yet reached the quality of multi-view stereo. Another problem is that these networks often do not generalize well beyond the specific image categories they have been trained on.

Light Field Capture: Several companies provide commercial solutions for capturing light fields for enabling motion parallax in VR, for example Lytro Immerge ${ }^{1}$ and OTOY ${ }^{2}$. However, these solutions require expensive hardware and target professional users while we are interested in methods that are suitable and affordable for casual users.

Normal Maps and Lighting: Some MVS algorithms use photoconsistency to explicitly compute normals during reconstruction [Furukawa and Ponce 2010; Galliani et al. 2015; Goesele et al. 2007; Schönberger et al. 2016]. While these normals agree well with the geometry, they are seldom of high enough quality to support lighting effects. One can improve the normals of 3D reconstruction using illumination cues, either as a post-process [Wu et al. 2011] or by explicitly incorporating them into the reconstruction process [Langguth et al. 2016].

\footnotetext{
https://www.lytro.com/

${ }^{2}$ https://home.otoy.com/otoy-demonstrates-first-ever-light-field-capture-for-vr/
} 

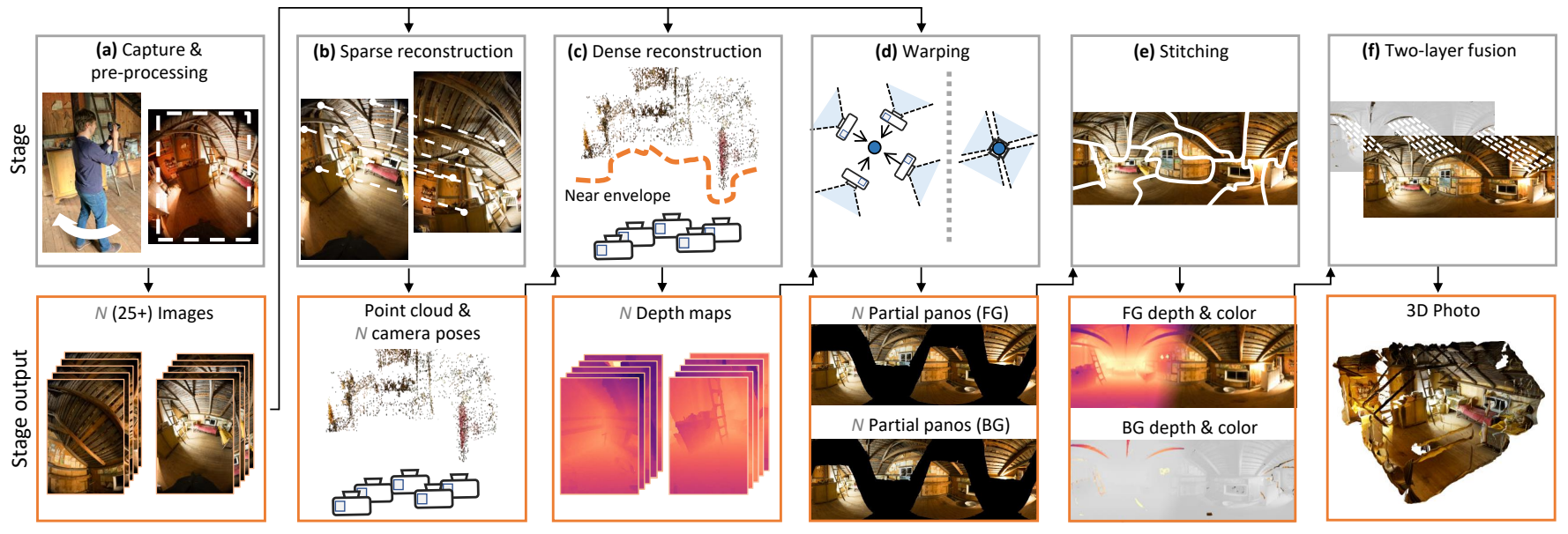

Fig. 2. A breakdown of the 3D photo reconstruction algorithm into its six stages, with corresponding inputs and outputs: (a) Capture and pre-processing, Sec. 4.1; (b) Sparse reconstruction, Sec. 4.2; (c) Dense reconstruction, Sec. 4.3; (d) Warping into a central panorama, Sec. 4.4.1; (e) Parallax-tolerant stitching, Sec. 4.4.2; (f) Two-layer fusion, Sec. 4.4.3.

In this paper, our goal is not to explicitly compute precise normals, but rather to generate plausible ones, sufficient for artistic lighting effects. Therefore, instead of estimating full lighting and reflectance distributions [Barron and Malik 2015; Debevec et al. 2004; Duchêne et al. 2015], we use perceptually plausible relighting techniques [Karsch et al. 2011; Khan et al. 2006] to hallucinate detail normals, which we combine with a smooth estimated base normal field.

\section{OVERVIEW}

One of our primary design goals is to make the 3D photo capture process easy for inexperienced users: the capture should be handheld, should not take too long, and should be captured with an existing, low-cost camera. These requirements influenced many of the algorithmic design decisions further down the pipeline.

The input to our reconstruction algorithm is a set of captured photos. We do not require them to be taken in any particular way, as long as they contain sufficient parallax and overlap to be registered by a standard structure from motion algorithm. In practice, it works best to capture while moving the camera on a sphere of about half arm's length radius. While we captured the images manually to produce the results in this paper, we envision a dedicated capture app could further simplify and speed up the process.

We represent a 3D photo in a single-viewpoint panoramic projection that is discretized into a pixel grid. While we use an equirectangular projection in our representation, other sensible choices, such as a cube map, would also be possible. Every pixel can hold up to two layers of "nodes" that store RGB radiance, normal vector, and depth values. This format resembles layered depth images [Shade et al. 1998]. However, in addition to the layered nodes, we also compute and store the connectivity between and among the two layers, in a manner similar to that of [Zitnick et al. 2004].

Our representation has several advantages: (1) the panoramic domain has an excellent resolution trade-off for rendering from a specific viewpoint, since it provides automatic level-of-detail where further away geometry is represented more coarsely than nearby features; (2) it can be stored compactly using standard image coding techniques and tiled for network delivery; (3) the two layers provide the ability to represent color and geometric details at disocclusions; (4) the connectivity enables converting the 3D photo into a dense mesh that can be rendered without gaps and easily simplified for low-end devices using standard techniques.

Our 3D photo reconstruction algorithm starts from a set of casually captured photos (Sec. 4.1, Fig. 2a) and uses an existing structure from motion algorithm to estimate the camera poses and a sparse geometric representation of the scene (Sec. 4.2, Fig. 2b). Our technical innovations concentrate in the following three stages:

Dense reconstruction (Sec. 4.3 / Fig. 2c). Taking the sparse reconstruction as a starting point, we first compute complete depth maps for the input images. We propose a novel prior, called the near envelope, that constrains the depth using a conservatively but tightly estimated lower bound. This prior results in highly improved reconstructions in our setting.

Parallax-tolerant stitching (Sec. 4.4.1-2 / Fig. 2d-e). The next goal is to merge the depth images into the final representation. We forwardwarp the depth images into the panoramic domain, for each image generating a front warp using a standard depth test and a back warp using an inverted depth test.

Two-Layer Fusion (Sec. 4.4.3 / Fig. 2f). Next, we merge these images into a front and back stitch, respectively, by solving a MRF problem. These two stitches are finally combined into the two-layer 3D photo representation using an algorithm that resolves connectivity among the layers, removes redundancies, and hallucinates new color and depth data in unobserved areas.

While our reconstructed 3D photo geometry is sufficient even for large viewpoint changes, when adding synthetic lighting and shading to the scene, the illusion is quickly destroyed, as the surface normal maps required for lighting effects are highly sensitive to even slight geometric errors. Similar to previous work [Khan et al. 2006] we resolve this issue by embossing intensity-hallucinated detail 
onto a coarse base normal map derived from smoothing the stitched geometry. The resulting normal map is not real but it contains plausible normals that enable artist-driven lighting effects (full-blown relighting is beyond the scope of this paper).

\section{3D PHOTO RECONSTRUCTION}

\subsection{Capture and Pre-processing}

Most of our scenes were captured with a mid-range Canon EOS $6 \mathrm{D}$ DSLR with a $180^{\circ}$ fisheye lens. To achieve nearly full $360 \times 180^{\circ}$ coverage, we captured two rings while pointing the camera slightly up and down, respectively. We fixed the exposure and captured about 25 input images on each ring. We preprocessed the RAW images in Adobe Lightroom to automatically white balance, denoise, and remove color fringing. We also cropped out the circular image region in the fisheye images, leaving about $160^{\circ} \times 107^{\circ}$ field-of-view (FOV).

We also experimented with capturing with a Samsung Galaxy S7 smart phone. Since the field-of-view is significantly narrower, we only captured partial panoramas, taking about $4 \times 4$ images on a grid. We used a single fixed exposure for each cell phone scene. This type of capture takes on the order of 30 seconds.

\subsection{Sparse Reconstruction}

We used the COLMAP structure from motion package [Schönberger et al. 2016] to recover the intrinsic and extrinsic camera parameters, the pose for each image, and a sparse point cloud representation of the scene geometry. We left most COLMAP options at their default, except for setting the camera model and initializing the focal length (see supplementary document for details). COLMAP also outputs undistorted rectilinear images of about $110^{\circ} \times 85^{\circ}$ and $65^{\circ} \times 50^{\circ} \mathrm{FOV}$ for the DSLR and cell phone camera, respectively, which we used for all subsequent processing steps.

\subsection{Dense Reconstruction}

Our first goal is to densify the reconstruction by computing a dense depth map for each input image using MVS. Unfortunately, our capture process breaks many common assumptions of these algorithms: (1) our baseline is narrow compared to the scene scale, making estimation of far geometry unreliable; (2) many of our scenes are not fully static and contain, for example, swaying trees and moving cars; (3) many scenes contain large textureless regions, such as sky or walls. These issues make MVS unreliable in the affected areas. In fact, most algorithms use some internal measure of confidence and drop pixels that are deemed unreliable, resulting in incomplete reconstructions. If they are forced to return a complete depth map, however, it is usually erroneous and/or noisy in those areas.

We solve this problem by introducing a novel near envelope reconstruction prior. The idea behind the prior is to propagate a conservative lower depth bound from confident to the less confident pixels. The prior effectively discards a large fraction of erroneous neardepth hypotheses from the cost volume, and causes the optimizer to reach a better solution.

4.3.1 Plane-sweep MVS Baseline. The baseline for our near envelope prior results is a state of the art plane-sweep MVS algorithm, which we describe briefly in this section. Please refer to the supplementary document for full implementation details.
Like prior work [Scharstein and Szeliski 2002], we treat depth estimation as an energy minimization problem. Let $i$ be a pixel and $c_{i}$ its color. We optimize the pixel depths $d_{i}$ by solving the following problem,

$$
\underset{d}{\operatorname{argmin}} \sum_{i} E_{\text {data }}(i)+\lambda_{\text {smooth }} \sum_{(i, j) \in \mathcal{N}} E_{\text {smooth }}(i, j),
$$

which consists of a unary data term and a pairwise smoothness term defined on a four-connected grid, $\lambda_{\text {smooth }}=0.25$ balances their contributions. The smoothness term is the product of a color- and a depth-difference cost,

$$
E_{\text {smooth }}(i, j)=w_{\text {color }}\left(c_{i}, c_{j}\right) w_{\text {depth }}\left(d_{i}, d_{j}\right),
$$

that encourages the depth map to be smooth wherever the image lacks texture (refer to the supplementary document for details). Our baseline data term consists only of a confidence-weighted photoconsistency term,

$$
E_{\text {data }}(i)=w_{\text {photo }}(i) E_{\text {photo }}(i),
$$

which measures the agreement in appearance between a pixel and its projection into multiple other images (full details for all terms are provided in the supplementary document).

We discretize the potential depth labels and use the plane-sweep stereo algorithm [Collins 1996] to build a cost volume with 220 depth hypotheses for each pixel. While this restricts us to reconstructing discrete depths without normals, it has the advantage that we can extract a globally optimized solution using an MRF solver, which can often recover plausible depth for textureless regions using its smoothness term. We optimize the MRF at a reduced resolution using the FastPD library [Komodakis and Tziritas 2007] for performance reasons. We then upscale the result to full resolution with a joint bilateral upsampling filter [Kopf et al. 2007], using a weighted median filter [Ma et al. 2013] instead of averaging to prevent introducing erroneous middle values at depths discontinuities.

4.3.2 Near Envelope. As mentioned before, most existing MVS algorithms, including the one described in Section 4.3.1, do not produce good results on our data. We have tried a variety of available commercial and academic algorithms (Sec. 6.2); Fig. 3 and Fig. 5b provide representative results. As can be seen, near-depth hypotheses are noisy, because these points are seen in fewer images and the photo-consistency measure is therefore less reliable. This makes MVS algorithms more likely to fall victim to common stereo pitfalls, such as: repeated structures in the scene, slight scene motion, or materials with view-dependent (shiny) appearance.

The idea behind the near envelope is to estimate a conservative but tight lower bound $n_{i}$ for the pixel depths at each pixel. We use this boundary to discourage nearby erroneous depths by augmenting the data term in Eq. 3 with an additional cost term,

$$
E_{\text {near }}(i)= \begin{cases}\lambda_{\text {near }} & \text { if } d_{i}<n_{i} \\ 0 & \text { otherwise }\end{cases}
$$

that penalizes reconstructing depths closer than the near envelope $\left(\lambda_{\text {near }}=1\right)$. The near envelope effectively prunes a large fraction of the cost volume, which makes it easier to extract a good solution.

To compute the near envelope, we first identify pixels with reliable depth estimates to serve as anchors (Fig. 4a-d). We propagate their depths to the remaining pixels using a color affinity smoothness 


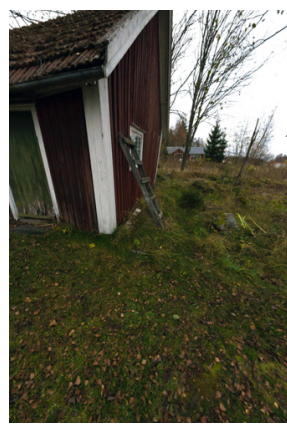

(a) Input view

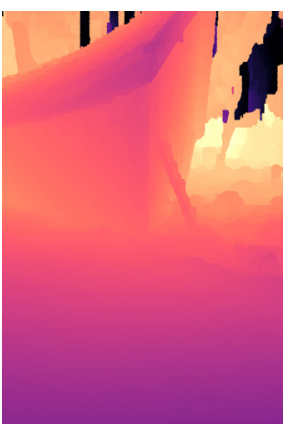

(b) MRF

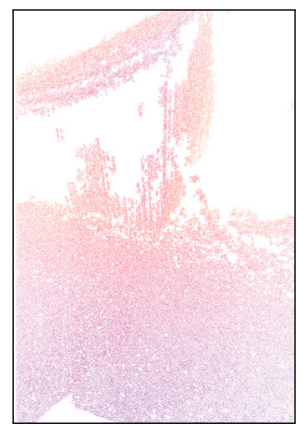

(c) PMVS

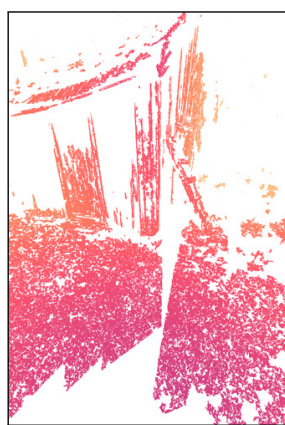

(d) MVE

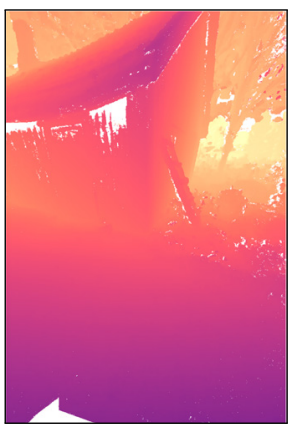

(e) COLMAP 2.1

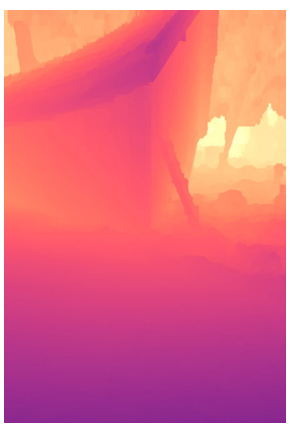

(f) $\mathrm{MRF}+\mathrm{NE}$ (ours)

Fig. 3. Existing state-of-the-art MVS algorithms (b-e) often produce incomplete and noisy depth maps for our casually captured data. Injecting our near-envelope prior into the MRF baseline algorithm shown in (b) produces superior results (f).

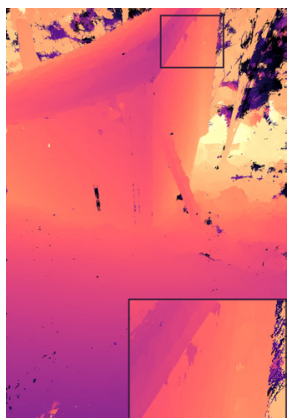

(a) WTA depth

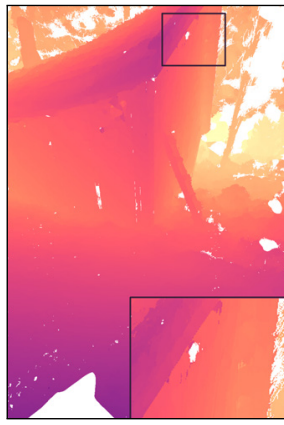

(b) Inconsistent visibility (GC filter)

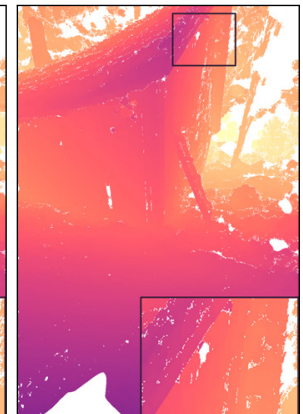

(c) Sparse noise (small median)

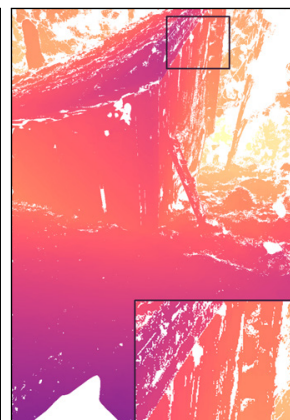

(d) Big floaters

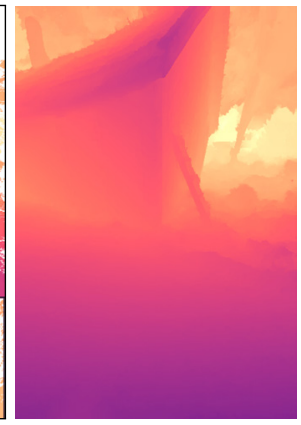

(e) Smooth propagation

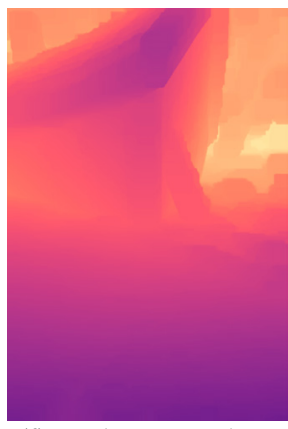

(f) Final near envelope (large bilateral median)

L_ Pruning unrelieable pixels from WTA depth

Fig. 4. The near envelope construction starts with a cheap to compute winner-takes-all stereo result (a). We use a series of filters (see text for details) to prune outliers. We fill the gaps that have formed by propagating the depths of the reliable pixels (e). The final envelope is obtained by applying a min-filter and scaling the result (f).

term (Fig. 4e), and obtain the near envelope by filtering the map and pulling it forward in depth (Fig. 4f).

Computing the anchor pixels: We start by computing a cheap "winner-takes-all" (WTA) stereo result (Fig. 4a) by dropping the smoothness term from Eq. 1 and independently computing the minimum of $E_{\text {data }}$ for each pixel. This quantity can be computed very fast, but the resulting depth maps are very noisy and contain a mixture of reliable and outlier depths (Fig. 4a). It is critical that the near envelope only be constructed from reliable pixels, otherwise it might either become ineffective due to being too lenient, or it might erroneously cut off true nearby objects, which then cannot be recovered by the final depth computation. Therefore, we wish to filter the outliers from the WTA depth maps. This is difficult, however, because we are faced with two conflicting objectives: (1) we want the filter to be conservative so it only lets through reliable pixels, but on the other hand (2) we need the filtered depth maps to be as complete as possible so we do not remove truly existing content.

We resolve this problem by applying a judiciously selected combination of pruning filters (Fig. 4b-4d). Each filter is focused on a different kind of outlier, but they are all designed to preserve nearly all reliable pixels, so that their combination achieves both objectives stated above.

The first step is to evaluate the geometric consistency (GC) measure [Wolff et al. 2016]. This boils down to examining each 3D point originating from a depth map against all other depth maps and checking whether it is consistent with surfaces implied by the other views. If the point is in conflict with the visibility in other depth maps or not in agreement with a sufficient number of other depth maps it is deemed inconsistent, and we remove the point (Fig. 4b, see Wolff et al.'s paper and the supplementary document for details).

We tune the filter carefully to avoid removing too many inliers. As a result, its output still contains some amount of outlier pixels that fall into two categories: sparse small noise regions (almost like salt-and-pepper noise) and larger floaters. These remaining outliers can be pruned using a median filter: a pixel is pruned if its depth is sufficiently different from the median filtered depth map (i.e., not within a factor of $[0.9,1.11])$.

Tuning the size of the median filter is problematic. A large filter removes thin structures, while a small filter cannot prune large floaters. Our solution is two split the filter into two steps. We first use a small median $(5 \times 5$ on a $900 \times 1350$ image $)$ to prune the sparse noise Fig. 4 c. To remove larger outliers we use a much larger $51 \times 51$ 

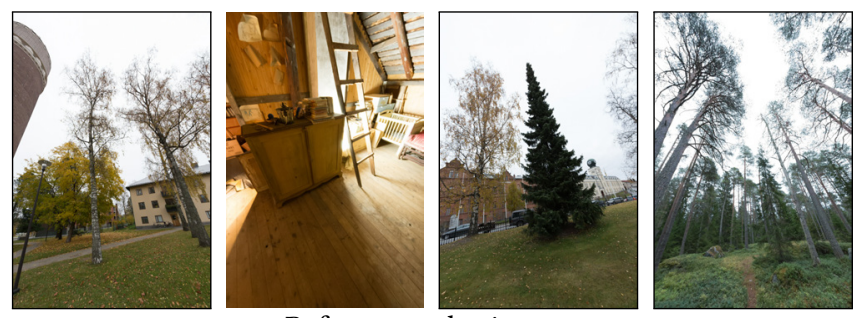

Reference color images
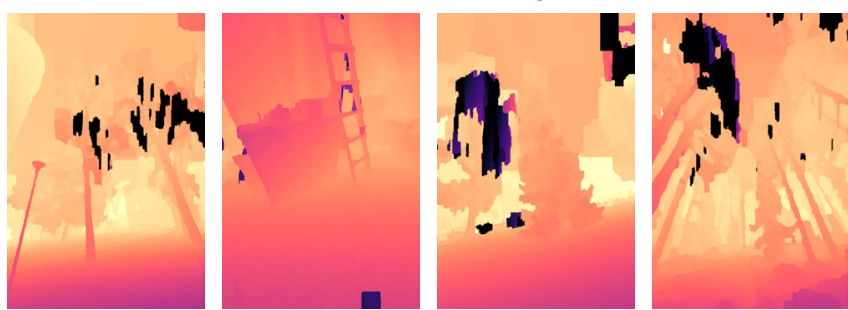

Plane-sweep + MRF without near envelope (standard)
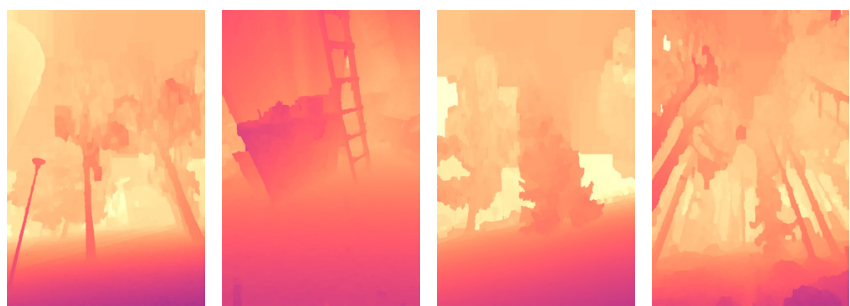

Plane-sweep + MRF with near envelope (our result)

Fig. 5. Comparison of plane sweep stereo without and with near envelope. Note the erroneous near-depths (dark colors) in the middle row. The supplementary material contains complete depth map result sets for all of our scenes.
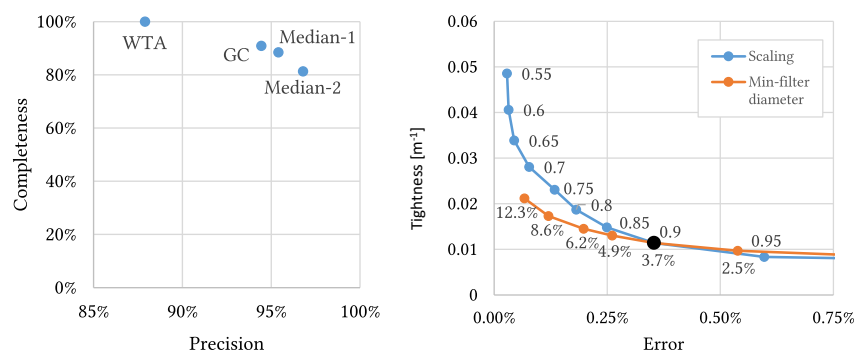

Fig. 6. Evaluating the near envelope construction using a set of ground truth depth maps from various scenes. Left: Completeness and precision plot for the outlier pruning stages. Right: Tightness and Error plots for the final near envelope. Please see the text for details.

median, but weigh it using a bilateral color term [Ma et al. 2013] $\left(\sigma_{c}=0.033\right)$ (Fig. 4d), which prevents the removal of thin structures.

Propagating the anchor depths: We spread the sparse anchor depths to the remaining pixels by solving a first-order Poisson system, similar to the one used in Levin et al.'s colorization algorithm [2004],

$$
\underset{x}{\operatorname{argmin}} \sum_{i} w_{i}\left(x_{i}-x_{i}^{\prime}\right)^{2}+\sum_{(i, j) \in \mathcal{N}} w_{i j}\left(x_{i}-x_{j}\right)^{2},
$$

where $x_{i}^{\prime}$ are the depths of the anchor pixels (where defined), and $x_{i}$ are the densely propagated depths we solve for. $w_{i j}=e^{-\left(c_{i}-c_{j}\right)^{2} / 2 \sigma_{e n v}^{2}}$ is the color based affinity term used by Levin et al. [2004] $\left(\sigma_{e n v}=0.1\right)$. $w_{i}=250 \sigma_{i}^{2}$ is a unary term that gives more weight to pixels in textured regions where MVS is known to be more reliable [Kopf et al. 2013], which we set to the variance $\sigma_{i}^{2}$ of the color in the surrounding $19 \times 19$ patch.

Computing the final near envelope: We make the propagated depths more conservative by multiplying them with a constant factor of 0.9 and subsequently applying a morphological minimum filter with diameter set to about $3 \%$ of the image diagonal.

Fig. 5 compares the results of the MVS algorithm from the previous section with and without the near envelope on a few representative images. In the supplementary material we provide the complete set of images for all our scenes, and also a comparison to other MVS algorithms.

Evaluation: To verify our near envelope construction algorithm, we generated a set of six ground truth depth maps across different scenes. We generated these by keeping only the most confident pixels from a COLMAP 2.1 reconstruction and manually inspected them for correctness.

For the WTA result as well as the three pruning stages (Figures Fig. 4a-d), we plot the completeness (percentage of pixels retained) as well as the precision (percentage of inliers among the retained pixels) (Fig. 6, left). The plots show that we retain a large fraction of pixels while eliminating nearly all outliers.

For the final near envelope (Fig. 4f), we evaluate the error (percentage of pixels where near envelope cuts actual content) as well as the tightness (average inverse distance between the near envelope and actual content). The sweeps for the min-filter size and scaling factor parameters in Fig. 6, right show that our default parameter (black dot) strikes a good balance between error and tightness.

\subsection{Parallax-tolerant Stitching and Two-layer Fusion}

In this section, we merge the depth maps into a panorama. Image alignment and stitching is a well studied problem [Szeliski 2006], but most methods assume that there is little parallax between the images. There are MVS algorithms that fuse depth maps (or directly compute a fused result) [Furukawa and Hernández 2015], but their output is not optimized for a specific viewpoint.

Our algorithm starts by warping all the depth maps into the panoramic domain (Sec. 4.4.1), then stitches a front surface and a back surface panorama (Sec. 4.4.2), and finally merges the two into a single two-layered mesh (Sec. 4.4.3).

4.4.1 Front and Back Surface Warping. We warp each depth map into a central panoramic image (using equirectangular projection) by triangulating it into a grid mesh and rendering it with the normal rasterization pipeline, letting the depth test select the front surface when multiple points fall onto the same panorama pixel. One problem with this simple approach is that long stretched triangles at depth discontinuities might obscure other good content, and we do not want to include them in the stitch.

We resolve this problem by blending the z-values of rasterized fragments with a stretch penalty $s \in[0,1]$ before the depth test, 


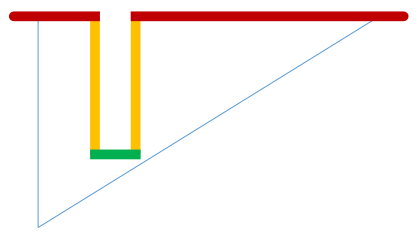

(a) front surfaces, normal depth test

(b) front surfaces, modified depth test

(c) back surfaces, inverted depth test

Fig. 7. We warp the depth maps into the panorama by rendering them with a special depth test to generate front and back surfaces for stitching. (a) highly stretched triangles at depth discontinuities can obscure other good content. (b) We use a modified depth test (see text) to prefer non-stretched triangles even if they are further away. (c) We obtain back surface warps by inverting the depth test.

$z^{\prime}=(z+s) / 2$. The division by 2 keeps the value $z^{\prime}$ in normalized clipping space. The stretch penalty,

$$
s=1-\min \left(\frac{\alpha}{\tau_{\text {stretch }}}, 1\right),
$$

considers the grazing angle $\alpha$ from the original viewpoint and penalizes small values below $\tau_{\text {stretch }}=1.66^{\circ}$, i.e., rays that are nearly parallel to the triangle surface. This modification pushes highly stretched triangles back, so potentially less stretched back surfaces can win over instead (Fig. 7b).

Since we are not just interested in only reconstructing the first visible surface, we generate a second back surface warp for each image. One possible way to generate this is depth peeling [Shade et al. 1998]; however, this method is best suited for accurate depth maps and did not perform well on our noisy estimates. We achieved more robust results, instead, by simply inverting the depth test, i.e. $z^{\prime \prime}=1-z^{\prime}$. This simple trick works well because when reprojecting depth maps to slightly new viewpoints, the resulting depth complexity rarely exceeds two layers.

4.4.2 Single Front and Back Layer Stitching. We are now ready to combine the warped color and depth maps from the previous section into stitched front and back surface panoramas with depth. This involves solving a discrete pixel labeling problem, where each pixel $i$ in the panorama chooses the label $\alpha_{i}$ from one of the warped sources. We use $c_{i}^{\alpha_{i}}$ to denote the color of $i$ from the source image $\alpha_{i}$ and use an analogous notation for other warped value maps (depth, stretch, etc.)

There are a number of different data and smoothness constraints that have to be considered and are sometimes conflicting. We first stitch the front, and subsequently the back-surface panorama by optimizing the following objective:

$$
\begin{aligned}
\underset{\left\{\alpha_{i}\right\}}{\operatorname{argmin}} \sum_{i} \underbrace{\lambda_{1} \Phi_{g c}(i)+\lambda_{2} \Phi_{\text {stretch }}(i)+\lambda_{3} \Phi_{\text {depth }}(i)}_{\text {Data terms }}+ \\
\sum_{i, j \in \mathcal{N}} \underbrace{\lambda_{4} \Psi_{\text {color }}(i, j)+\lambda_{5} \Psi_{\text {disp }}(i, j)}_{\text {Smoothness terms }} .
\end{aligned}
$$

The geometric consistency term $\Phi_{g c}$ is a binary function set to 1 for pixels that fail the test [Wolff et al. 2016] (see supplementary material), and 0 otherwise. The triangle stretch term $\Phi_{\text {stretch }}$ discourages selecting pixels from long "rubber sheet" triangles (using the stretch penalty defined in Eq. 6):

$$
\Phi_{\text {stretch }}(i)=-\log s_{i}^{\alpha_{i}} .
$$

When stitching front surfaces, we use the depth data terms $\Phi_{\text {depth }}$ to encourage depths that are consistent among many views. Similar to the GC filter, we project the 3D point for each pixel into all other depth maps and check whether the projected point's depth and the depth map value are similar. We count the number $n_{i}$ of other depth maps that are consistent in this manner, and set the depth data term:

$$
\Phi_{\text {depth }}(i)=1-\min \left(\frac{n_{i}}{\tau_{\text {overlap }}}, 1\right) .
$$

$\tau_{\text {overlap }}=5$ specifies how many depth maps need to agree before we consider a pixel to be reliable.

When stitching back surfaces, we use a different depth term that discourages selecting pixels that are closer than the front stitch:

$$
\Phi_{\text {depth }}(i)= \begin{cases}10 & \text { if } d_{i}^{\alpha_{i}}<0.95 d_{i}^{\text {front }} \\ 0 & \text { otherwise. }\end{cases}
$$

The color smoothness term $\Psi_{\text {color }}$ is a truncated version of the seam-hiding pairwise cost from the GraphCut Textures paper [Kwatra et al. 2003]:

$$
\Psi_{\text {color }}(i, j)=\min \left(\left\|c_{i}^{\alpha_{i}}-c_{i}^{\alpha_{j}}\right\|_{2}^{2}, \tau_{c}\right)+\min \left(\left\|c_{j}^{\alpha_{i}}-c_{j}^{\alpha_{j}}\right\|_{2}^{2}, \tau_{c}\right),
$$

and the depth smoothness term $\Psi_{\text {disp }}$ is a similar truncated term (working with disparities):

$$
\Psi_{d i s p}(i, j)=\min \left(\left|\frac{1}{d_{i}^{\alpha_{i}}}-\frac{1}{d_{i}^{\alpha_{j}}}\right|, \tau_{d}\right)+\min \left(\left|\frac{1}{d_{j}^{\alpha_{i}}}-\frac{1}{d_{j}^{\alpha_{j}}}\right|, \tau_{d}\right) .
$$

The depth $d$ is normalized to metric units, and we use the truncation thresholds $\tau_{c}=0.05, \tau_{d}=0.05 \mathrm{~m}^{-1}$.

We set the following balancing coefficients,

$$
\lambda_{1}=200, \lambda_{2}=1000, \lambda_{3}=100, \lambda_{4}=200, \lambda_{5}=100,
$$

and solve the labeling problems at a reduced $512 \times 256$ resolution (to achieve a reasonable performance) using the alpha expansion algorithm [Kolmogorov and Zabih 2004]. We then upsample the resulting label map to full resolution $(8192 \times 4096$ pixels) using a simple PatchMatch-based upsampling algorithm [Besse et al. 2014].

4.4.3 Two-layer Merging. Now that we have obtained a front and back stitch, our next goal is to fuse them into the final two-layer representation, to produce a light-weight mesh representation of the scene that is easy to render on a wide variety of devices. Compared to rendering both layers separately, the two-layer fused mesh provides several benefits by (1) resolving which pixels should be connected as part of the same surfaces and where there should be gaps (2) removing color fringes and allows for seamless texture filtering across layers, (3) identifying and discarding redundant parts of the background layer, (4) hallucinating unseen geometry by extending the background layer.

We represent the two-layer panorama as a graph. Each pixel $i$ in the panorama has up to two nodes that represent the foreground and background layers; if they exist, we denote these nodes as $f_{i}$ and $b_{i}$. Each node $n$ has a depth value $d(n)$ and a foreground / background label $l(n) \in\{F, B\}$.

We start by generating for both front and back stitches, fully 4connected but disjoint grid-graphs (Fig. 9, top). Each node is assigned a depth and label according to the stitch it is drawn from. 


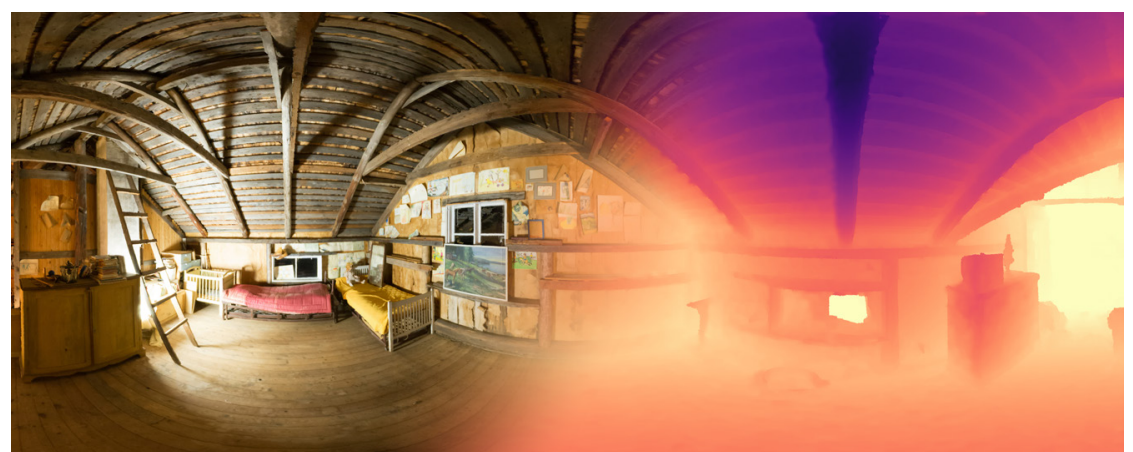

(a) Front color-and-depth panorama

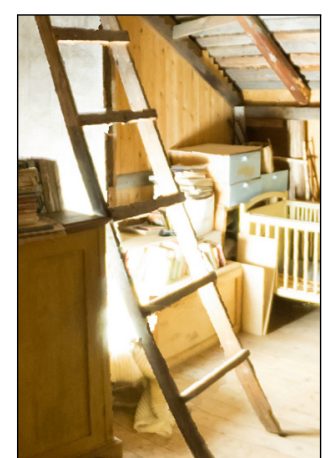

(b) Front detail

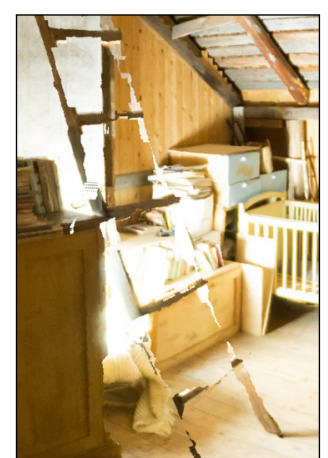

(c) Back detail

Fig. 8. Our algorithm first stitches a color-and-depth panorama for the front-surfaces (a). Its depth is used as constraint, when subsequently stitching the back-surface panorama (c, only detail shown). Note the eroded foreground objects when comparing the back and front details (b-c).

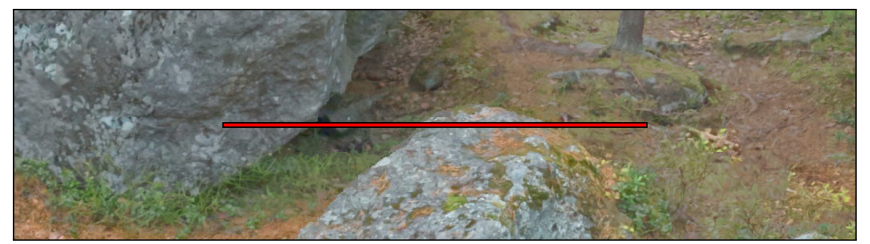

Input: color

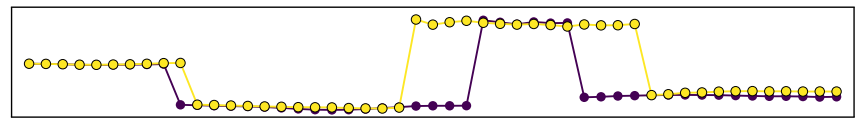

Input: initial fully connected $f$ and $b$ graphs

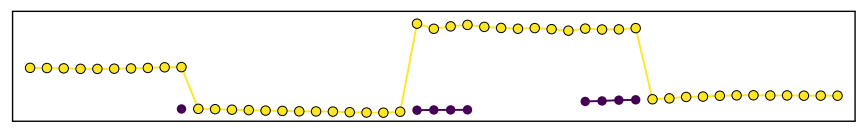

Step 1: remove redundant $b$ nodes

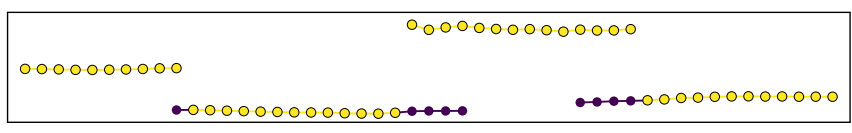

Step 2: recompute connectivity

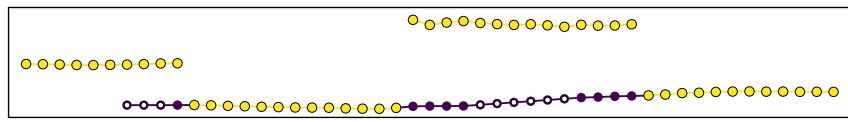

Step 3: expand $b$, hallucinate depth and color

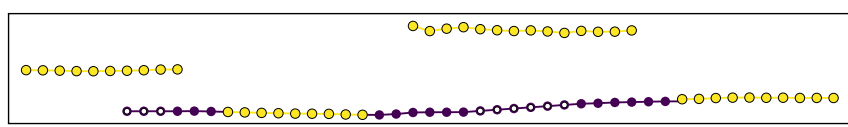

Step 4: propagate $B$ label onto $f$ nodes to remove color fringes

Fig. 9. Fusing the front and back stitches into a single two-layer graph representation. The diagrams show the front and back nodes for the highlighted scanline. Nodes with $F$ and $B$ labels are drawn in yellow and purple, respectively. Nodes with hallucinated depth and color are outlined.

Note that these graphs contain redundant coverage of some scene objects, e.g., the inner core of the rocks in Fig. 9. This is fully intentional and we take advantage of it to remove color fringes around

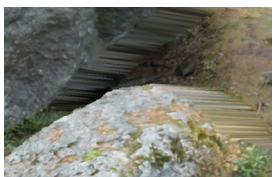

Initial graph

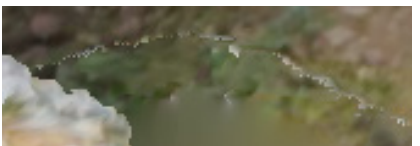

Before color fringe removal

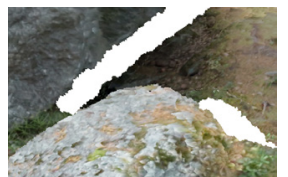

Before expansion

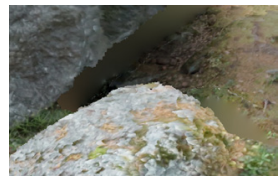

After expansion

Final result

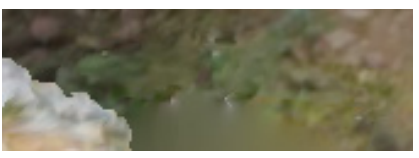

Fig. 10. Steps of the two-layer fusion algorithm. Compare with the diagrams in Fig. 9.

depth continuities further below. However, for now, we remove the redundancies by removing all the $b_{i}$ nodes that are too similar to their $f_{i}$ counterparts, i.e., $d\left(f_{i}\right) / d\left(b_{i}\right)<\tau_{\text {dratio }}=0.75$ (step 1 in Fig. 9).

The graphs are not redundant anymore, but now the $b$ graph contains many isolated components, and the $f$ graph contains long connections across discontinuities. We recompute the connectivity (step 2 in Fig. 9) as follows. For each pair of horizontally or vertically neighboring pixels we consider all combinations of $f$ and $b$ nodes and sort them by their depth ratio, most similar first. Then we connect the most similar pair if the depth ratio is above $\tau_{\text {dratio. }}$. If there is another pair that can be connected without intersecting with the previous one, we connect that as well. Now we have a well-connected two-layer graph.

For small viewpoint changes, the back layer provides some extra content to fill gaps, but large translations can still reveal holes. To improve this, we expand the back layer by hallucinating depth and color (step 3 in Fig. 9) in an iterative fashion, one pixel ring at a time. At each iteration, we identify $b$ and $f$ nodes that are not connected in one direction. We tentatively create a new candidate neighbor node for these pixels and set their depths and colors to the average of the nodes that spawned them. We keep candidate nodes only if they are not colliding with already existing nodes (using $\tau_{\text {dratio }}$ ), and if they become connected to the nodes that spawned them. 


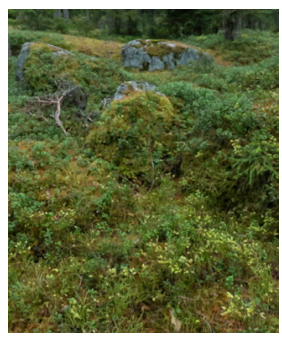

(a) Unlit rendering

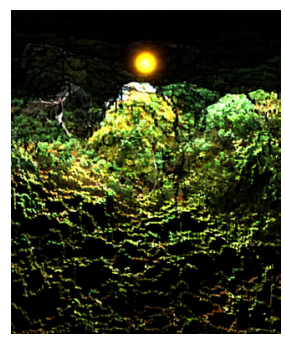

(b) Lit with forward-diff normals

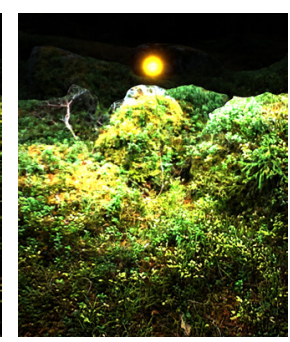

(c) Lit with our normals

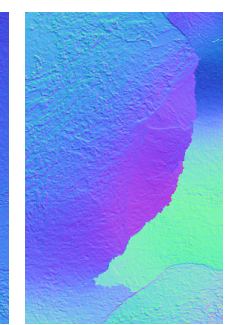

(g) Final normals

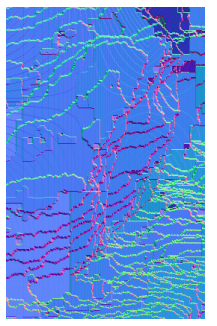

(d) Forward-diff normals

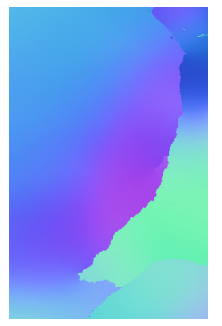

(e) Smoothed base

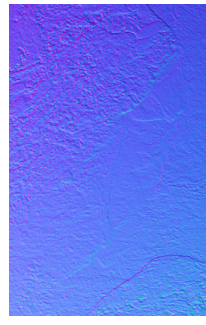

(f) Image-based details
Table 1. Approximate timings for the 3D photo reconstruction algorithm stages for a whole scene in h:mm.

\begin{tabular}{lr} 
Stage & Timing \\
\hline Sparse reconstruction (Sec. 4.2) & $0: 40$ \\
Dense reconstruction (Sec. 4.3) & $3: 00$ \\
Front and Back Warping (Sec. 4.4.1) & $0: 15$ \\
Front and Back Stitching (Sec. 4.4.2) & $0: 30$ \\
Two-layer Fusion (Sec. 4.4.3) & $0: 30$ \\
Normal Map Estimation (Sec. 5) & $0: 20$ \\
\hline Total & $\mathbf{5 : 1 5}$
\end{tabular}

by applying the guided filter to the depth normals, and $n_{i}$ be the normal obtained from the image-based estimation method. Let $R_{S}$ be a local coordinate frame for the image-based normal. We obtain it by setting the first row to a vector pointing radially outward,

$$
R_{s, 0}=(\sin \theta \cos \phi, \cos \theta, \sin \theta \sin \phi)
$$

and the other rows through cross products with the world up vector $\mathbf{w}_{\text {up }}$,

$$
R_{s, 1}=\frac{R_{s, 0} \times \mathbf{w}_{u p}}{\left\|R_{s, 0} \times \mathbf{w}_{u p}\right\|}, R_{s, 2}=R_{s, 0} \times R_{s, 1} .
$$
are not sufficient for convincing lighting effects (b). We improve the normal map by combining a smoothed version (e) with surface details extracted from the color image alone (f). The combined normal map (g) is fake but plausible, and enables interesting artistic lighting effects (c).

\section{LIGHTING}

The final step of our reconstruction pipeline is to compute a normal map to enable simple artist-driven lighting effects (Fig. 11). Note that we are merely interested in normals that look plausible and are good enough for simple lighting effects; full-blown relighting is beyond the scope of this paper.

Adding lights to a synthetic scene requires estimating the normal vector at every scene point. Our depth looks convincing when rendered with diffuse texture. However, it contains too many artifacts when used for relighting (Fig. 11b). We resolve this by synthesizing a normal map that is a combination of a smooth base map with the right slopes and a detail map that is hallucinated from the color image data (Fig. 11e-g).

The base normal map should be piece-wise smooth but discontinuous at depth edges and contain the correct surface slopes. We compute this map by filtering normals obtained by taking forward differences with a linear system similar to Eq. 5, except the data constraints $x_{i}^{\prime}$ are defined everywhere and the smoothness weights $w_{i j}$ are binary weights, set to zero at the discontinuities identified in the post-processing in Sec. 4.4.2, and set to one everywhere else.

We hallucinate the details map from the luminance image using the method of Khan et al. [2006]. Their method estimates a normal map by hallucinating a depth map from just the image data, assuming surface depth is inversely related to image intensity. While the depth generated in this manner is highly approximate, it is consistent with the image data and provides a surprisingly effective means for recovering geometric detail variations.

We combine these two cues as follows. For a given pixel with azimuthal and polar angles $(\theta, \phi)$, let $n_{f}$ be the normal obtained
We define a similar coordinate frame $R_{f}$ for the filtered depth normal, setting $R_{f, 0}=-n_{f}$ and the other rows analogously to Eq. 15 .

We can now transfer the details as follows:

$$
n_{c}=R_{f}^{-1} R_{s} n_{i}
$$

Since often sky pixels are reconstructed at an arbitrary depth, we use a sky detector [Schmitt and Priese 2009] on the stitched panorama to selectively disable lighting in the sky (by setting the normals to zero).

\section{RESULTS AND EVALUATION}

We have captured and reconstructed a number of 3D photos that can be seen in Fig. 12, as well as the accompanying video and supplemenand 5 with cell phone cameras, as described in Sec. 4.1. The scenes span man-made and natural environments, indoors and outdoors, as well as full $360^{\circ} \times 180^{\circ}$ and partial coverage. They contain many elements that are difficult to reconstruct, such as water surfaces, swaying trees, and moving cars and people.

Like other end-to-end reconstruction systems, our system depends on many parameters. We tuned each component individually, following the data flow. For each component, we identified opposing artifacts in a subset of the scenes (e.g., thin objects vs. textureless areas), and performed a parameter sweep to find values that balance both kinds of artifacts. All results provided in this paper and supplementary material and video were created using the same parameter settings, except lens calibration.

We have experimented with a variety of geometry-aware and lighting effects, including flooding a scene with virtual water, adding a moving fairy light, and a midnight light show. Figs. 1 and 11 show some examples; more can be seen in the accompanying materials. tary material. 14 of these scenes were captured with a DSLR camera 

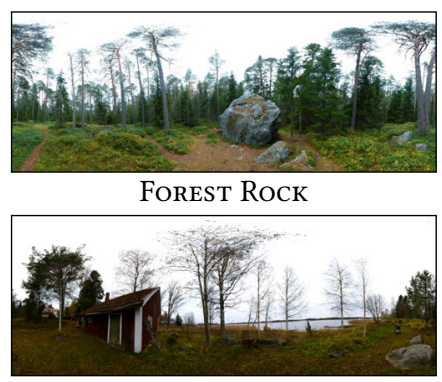

BoAt Shed

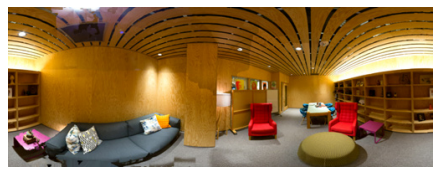

LIBRARY

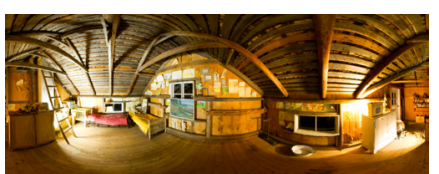

Creepy Attic

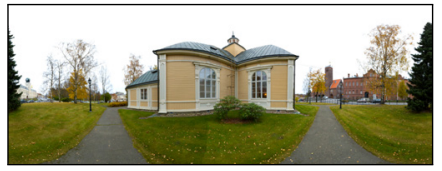

$\mathrm{CHURCH}$

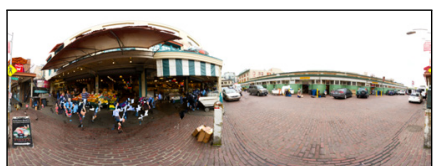

Pike Place

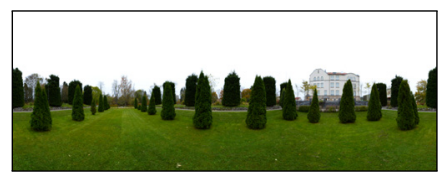
GyMNASIUM

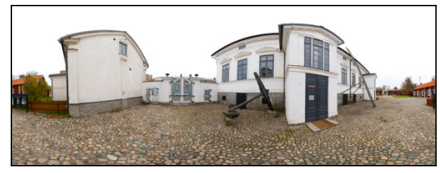

JAKOBSTAD MUSEUM

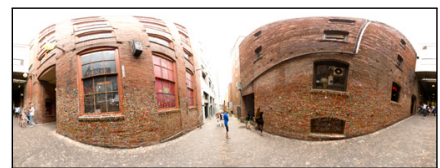

GUM WALL

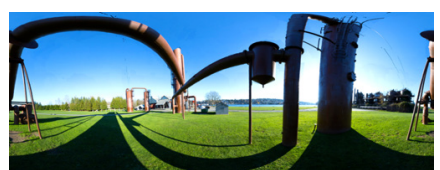

Gas Works Park

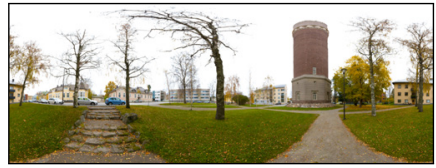

WATER TOWER

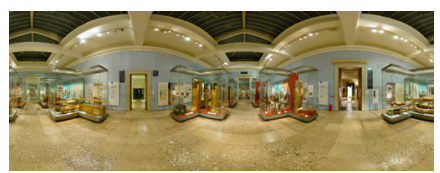

British Museum $360^{\circ} \times 180^{\circ}$ scenes captured with DSLR cameras

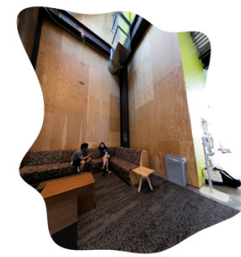

SofA

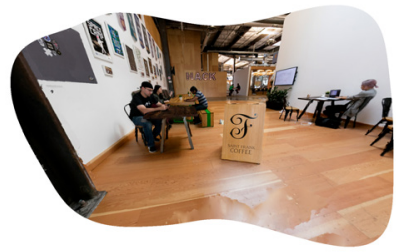

CAFE

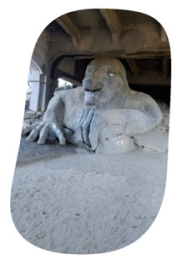

TROLL

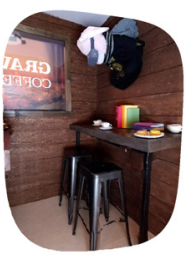

Gravity

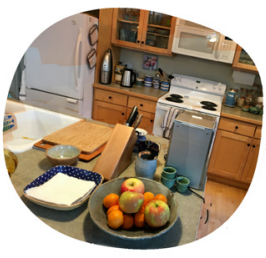

KITCHEN

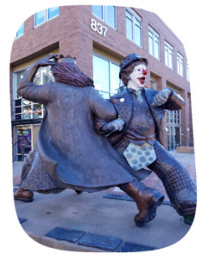

Clowns

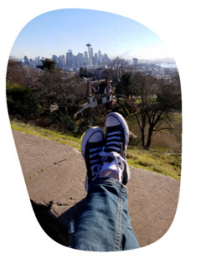

KeRry PARK

— Partial scenes captured with DSLR cameras $\_$

Partial scenes captured with cell phone cameras

Fig. 12. Some 3D photos we have captured with DSLR and phone cameras.

\subsection{Performance}

All of the 3D photos were reconstructed on a single 6-Core Intel Xeon PC with an NVIDIA Titan X GPU and 256 GB of memory. Table 1 lists timings for a representative DSLR capture.

While our current implementation is slow, we note that there is significant room for improvement. The two bottlenecks are the sparse and the dense scene reconstruction steps, and each can be sped up significantly. A SLAM algorithm would provide a much faster alternative to the slow structure from motion (SfM) algorithm, and it could even improve the results since SLAM is optimized for sequentially captured images while SfM is designed for unstructured wide-baseline input. The MVS reconstruction is by far the slowest step in our pipeline, but it could be trivially parallelized, since each image is reconstructed independently from the others.

\subsection{Comparative Evaluation}

We have made extensive quantitative and qualitative experiments comparing our system with the following academic and commercial end-to-end reconstruction systems:

PMVS: We reconstruct a semi-dense point cloud with PMVS [Furukawa and Ponce 2010] and then use Screened Poisson Surface Reconstruction [Kazhdan and Hoppe 2013] to create a watertight surface.
MVE: We use the Multi-View Environment [Fuhrmann et al. 2014] implementations of Goesele et al.'s [2007] semi-dense reconstruction and Floating Scale Surface Reconstruction for texturing [Fuhrmann and Goesele 2014].

GDMR: The Global, Dense Multiscale Reconstruction method [Ummenhofer and Brox 2015] starts from the same semi-dense reconstruction as MVE, but provides an alternative surface reconstruction and texturing method.

COLMAP: COLMAP 2.1 [Schönberger et al. 2016] provides an end-to-end pipeline for sparse reconstruction, depth map computation, and fusion.

PhotoScan: Agisoft PhotoScan ${ }^{3}$ is a commercial end-to-end reconstruction pipeline.

Capturing Reality: This $^{4}$ is another state-of-the-art end-to-end reconstruction pipeline.

We use the publicly available implementations for each system. All systems have in common that they initially perform or require as input a sparse reconstruction, which is subsequently densified. To provide a fair comparison, we used the same sparse reconstruction (computed with COLMAP) for all systems, including ours.

Some of the methods produce impressive geometric reconstructions but only relatively coarse textures. For this reason we experimented with the following alternative texturing methods, in addition to whatever native textures each system produces:

\footnotetext{
${ }^{3}$ http://www.agisoft.com/

${ }^{4}$ https://www.capturingreality.com/
} 


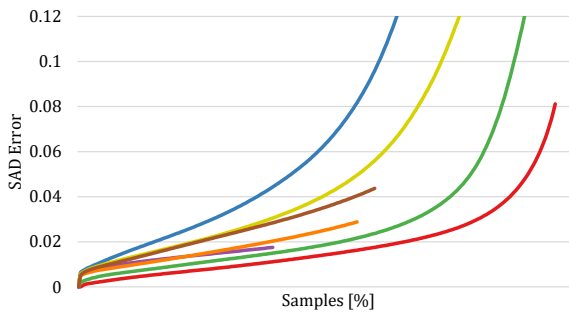

Native Textures

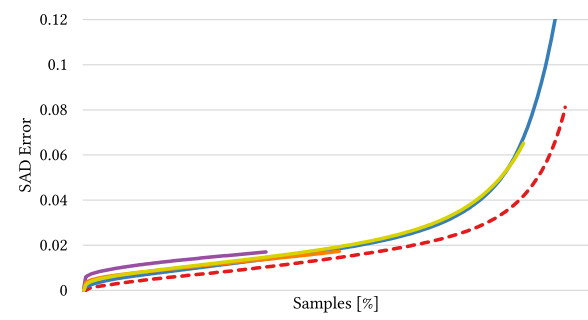

TexRecon Textures [Waechter et al. 2014]

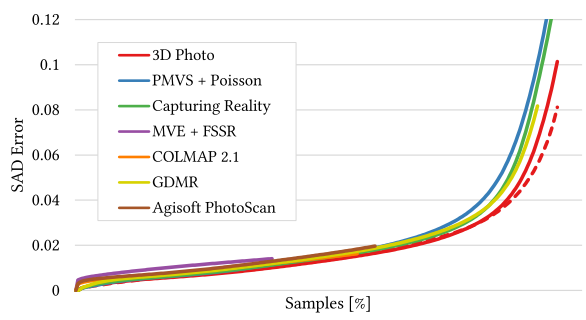

Unstructured Lumigraph [Buehler et al. 2001]

Fig. 13. Plotting the average virtual rephotography error against completeness for different reconstruction and texturing methods. The dotted red line represents our full system with native textures, i.e., is identical to the solid line in the left plot.

TexRecon [Waechter et al. 2014]: This method produces a highquality texture atlas for a given 3D model and images that are registered against this model.

Unstructured Lumigraph Rendering [Buehler et al. 2001]: This method uses the 3D model as a geometric proxy for ImageBased Rendering. For each pixel we blend the two top-scoring images to avoid ghosting.

\subsection{Quantitative Evaluation}

We use Virtual Rephotography [Waechter et al. 2017] to provide a quantitative comparison against the systems mentioned before. This is a unified evaluation method for end-to-end reconstruction-andrendering methods. Its idea is to render the reconstruction from the exact same viewpoint as each input image ("virtual rephotos"), and compare the results against the ground truth images with respect to completeness and visual error.

Since the texture resolution of the methods differ, we render results at about $20 \%$ of the input image resolution ( 900 pixels width). For each pixel, we evaluate the minimum sum of absolute differences (SAD) error within a shiftable window of \pm 2 pixels.

Fig. 13 provides error and completeness scores aggregated over all 15 scenes for each reconstruction and the three alternative ways of texturing them, and Fig. 14 provides a visual result for an example image. In the supplementary material, we provide a more fine-grained break-down of these results.

\subsection{Qualitative Evaluation}

To help the reader evaluate the visual quality of the results, we provide a full set of videos with scripted camera paths for all scenes and all methods in the supplementary material, and a web page for convenient side-by-side visual inspection.

A major limitation of most systems we compared against is that they do not produce complete results. Most methods do not reconstruct far regions, due to reliability thresholds in the depth estimation. Another source of missing regions are texture-less areas, where matching is ambiguous. Our technique produces far more complete results and relies on MRF-based smoothing to fill in unreliably or ambiguous regions.

MRF smoothing also helps in regions with highly complex geometry, where systems without smoothing (e.g., COLMAP 2.1) produce noisy results.

Some of the systems produce only relatively coarse vertex color textures. TexRecon [Waechter et al. 2014] does a good job in improving the texturing, at the expense of making the results a bit less complete, since it removes uncertain triangles. Another alternative is image-based rendering [Buehler et al. 2001]. While IBR is very good at optimizing the reprojection error, it has several downsides: the display is less stable as texturing is view-dependent, leading to visibly moving texture seams when moving the camera. Another disadvantage of IBR is that it is far more expensive than native texturing in terms of data size (all source images need to be retrieved and retained in memory) as well as performance (shading).

\subsection{Stitching Evaluation}

In Fig. 15, we show the effect of running our system without the near envelope and without the GC filter (see the supplementary material for more results). The near envelope provides a substantial improvement in many scenes. Without it, we often see many floaters (dark spots in Fig. 15a) that lead to visual artifacts (Fig. 15b). Disabling the GC filter yields more edge fattening in many scenes, though its impact is less dramatic than the near envelope.

\subsection{Limitations}

Our system has some important limitations, which are inherited from the underlying sparse and dense reconstruction algorithms. The stereo algorithm may fail to reconstruct shiny and reflective surfaces (e.g., British Museum), as well as dynamically moving objects such as people (e.g., Pike Place, Gum Wall). This might might lead to erroneously estimated depth. Often, our subsequent processing stages can fix these problems, e.g., the stitching step deals well with geometric outliers in single depth maps. But in some of our results, small artifacts can still be found under close inspection (see supplemental videos). In partially captured scenes some artifacts may appear near boundaries where fewer input images observed the scene.

Another minor limitation is that our current implementation does not perform any blending, which sometimes leads to visible color discontinuities (e.g., Library, Kitchen). It would be relatively straight-forward to add color blending and even depth-blending [Luo et al. 2012] to our system.

A limitation shared with most modern 3D reconstruction systems is the dependency on many parameters. We found, however, that besides lens calibration parameters, all other parameter settings generalize well. In fact, we used the same settings for all scenes shown throughout the submission, even though they are produced with very different types of cameras.

Another important limitation is that our approach cannot produce $3 \mathrm{D}$ videos, because the casual capture process requires moving a 


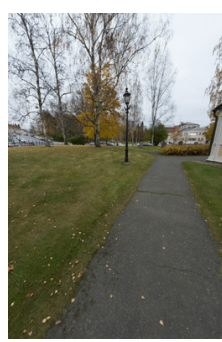

(a) Reference

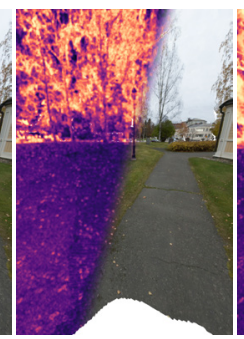

(b) Our result

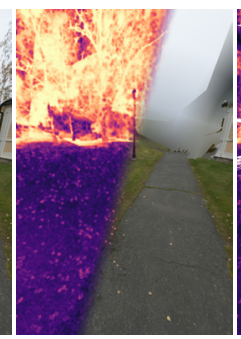

(c) Capturing reality

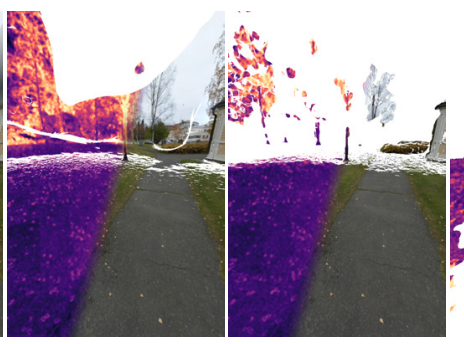

(d) GDMR \&

TexRecon (e) COLMAP $2.1 \&$

TexRecon

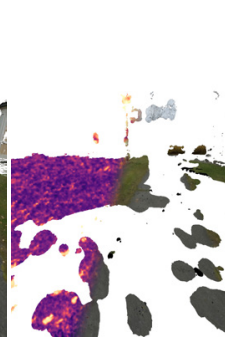

(f) Photoscan

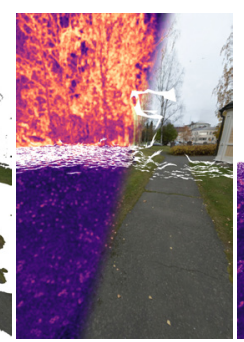

(g) PMVS \& TexRecon

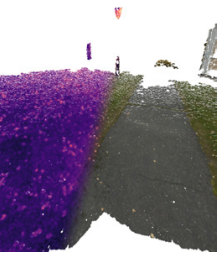

(h) MVE \&

TexRecon

Fig. 14. Rendered results and corresponding rephotography errors in the Church scene. Dark regions have lower error.

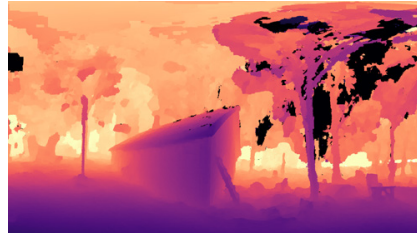

(a) Depth without near-envelope

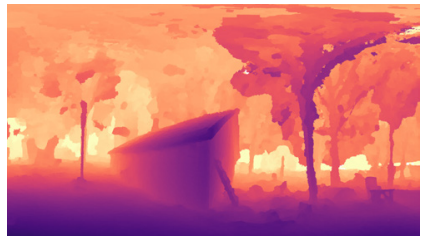

(c) Depth with near-envelope

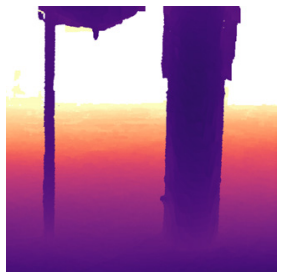

(e) Stitched without GC cost

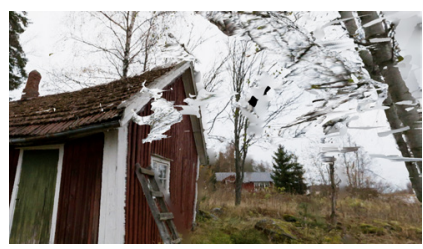

(b) Render without near envelope

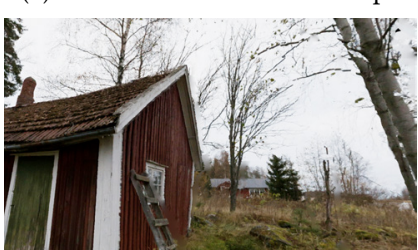

(d) Render with near envelope

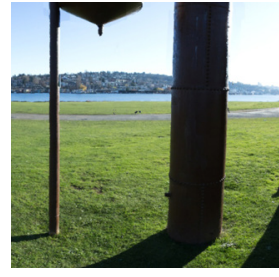

(f) Stitched colors

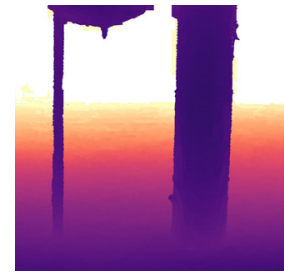

(g) Stitched with GC cost
Fig. 15. Showing the effect of running our system without the near envelope prior (a-d), as well as without GC cost in the stitcher (e-g). Running without near envelope yields many floaters that result in visual artifacts. The effect of disabling the GC cost is more subtle, it results in more edge fattening.

single camera in space. It would be possible, however, to apply our algorithm to structured input captured by a multi-camera rig. This is an interesting avenue for future work.

\section{CONCLUSIONS}

In this paper, we have developed a novel system to construct seamless two-layer 3D photographs from sequences of casually acquired photographs. Our work builds on a strong foundation in sparse and dense MVS algorithms, with enhanced results due to our novel near envelope cost volume prior. Our parallax-tolerant stitching algorithm further removes many outlier depth artifacts. It produces front and back surface panoramas with well-reconstructed depth edges, because it starts from depth maps whose edges are aligned to the reference image color edges. Our fusion algorithm fuses the front and back panoramas into a single two-layer 3D photo.

The consequence of all these technical innovations is that we can apply our algorithm to casually captured input images that violate many common assumptions of MVS algorithms. Most of our scenes contain a variety of difficult to reconstruct elements, such as dynamic objects (people, swaying trees), shiny materials (lake surface, windows), and textureless surfaces (sky, walls). The fact that we nevertheless succeed in reconstructing relatively artifact-free scenes speaks for the robustness of our approach.

\section{ACKNOWLEDGEMENTS}

The authors would like to thank Scott Wehrwein, Tianfan Xue, Jan-Michael Frahm and Kevin Matzen for helpful comments and discussion.

\section{REFERENCES}

Robert Anderson, David Gallup, Jonathan T. Barron, Janne Kontkanen, Noah Snavely, Carlos Hernandez Esteban, Sameer Agarwal, and Steven M. Seitz. 2016. Jump: Virtual Reality Video. ACM Transactions on Graphics 35, 6 (2016).

Jonathan T. Barron and Jitendra Malik. 2015. Shape, Illumination, and Reflectance from Shading. IEEE Trans. Pattern Anal. Mach. Intell. 37, 8 (2015), 1670-1687.

Frederic Besse, Carsten Rother, Andrew Fitzgibbon, and Jan Kautz. 2014. PMBP: PatchMatch Belief Propagation for Correspondence Field Estimation. Int. F. Comput. Vision 110, 1 (2014), 2-13.

Aaron F. Bobick and Stephen S. Intille. 1999. Large Occlusion Stereo. International Journal of Computer Vision 33, 3 (1999), 181-200.

Chris Buehler, Michael Bosse, Leonard McMillan, Steven Gortler, and Michael Cohen. 2001. Unstructured Lumigraph Rendering. (2001), 425-432.

Gaurav Chaurasia, Sylvain Duchene, Olga Sorkine-Hornung, and George Drettakis 2013. Depth Synthesis and Local Warps for Plausible Image-based Navigation. ACM Trans. Graph. 32, 3 (2013), 30:1-30:12.

Robert T. Collins. 1996. A space-sweep approach to true multi-image matching. In IEEE Conference on Computer Vision and Pattern Recognition (CVPR 1996). 358-363.

Paul Debevec, Chris Tchou, Andrew Gardner, Tim Hawkins, Charis Poullis, Jessi Stumpfel, Andrew Jones, Nathaniel Yun, Per Einarsson, Therese Lundgren, Marcos Fajardo, and Philippe Martinez. 2004. Estimating Surface Reflectance Properties of a Complex Scene under Captured Natural Illumination. ICT Technical Report ICT TR 062004 (2004).

Paul E. Debevec, Camillo J. Taylor, and Jitendra Malik. 1996. Modeling and Rendering Architecture from Photographs: A Hybrid Geometry- and Image-based Approach. In Proceedings of the 23rd Annual Conference on Computer Graphics and Interactive Techniques (SIGGRAPH '96). ACM, New York, NY, USA, 11-20. https://doi.org/10. $1145 / 237170.237191$

Sylvain Duchêne, Clement Riant, Gaurav Chaurasia, Jorge Lopez-Moreno, Pierre-Yves Laffont, Stefan Popov, Adrien Bousseau, and George Drettakis. 2015. Multi-View Intrinsic Images of Outdoors Scenes with an Application to Relighting. ACM Transactions on Graphics (2015).

David Eigen, Christian Puhrsch, and Rob Fergus. 2014. Depth Map Prediction from a Single Image Using a Multi-scale Deep Network. Proceedings of the 27th International Conference on Neural Information Processing Systems (NIPS) (2014), 2366-2374. 
Jakob Engel, Vladlen Koltun, and Daniel Cremers. 2016. Direct Sparse Odometry. arXiv:1607.02565 (2016).

Facebook. 2016. Facebook Surround 360. https://facebook360.fb.com/ facebook-surround-360/. (2016). Accessed: 2016-12-26.

John Flynn, Ivan Neulander, James Philbin, and Noah Snavely. 2016. DeepStereo Learning to Predict New Views From the World's Imagery. The IEEE Conference on Computer Vision and Pattern Recognition (CVPR) (2016).

Simon Fuhrmann and Michael Goesele. 2014. Floating Scale Surface Reconstruction. ACM Trans. Graph. 33, 4 (2014), article no. 46

Simon Fuhrmann, Fabian Langguth, and Michael Goesele. 2014. MVE: A Multi-view Reconstruction Environment. Proceedings of the Eurographics Workshop on Graphics and Cultural Heritage (GCH'14) (2014), 11-18.

Yasutaka Furukawa and Carlos Hernández. 2015. Multi-View Stereo: A Tutorial. Foundations and Trends. in Computer Graphics and Vision 9, 1-2 (2015), 1-148.

Yasutaka Furukawa and Jean Ponce. 2010. Accurate, Dense, and Robust Multiview Stereopsis. IEEE Trans. Pattern Anal. Mach. Intell. 32, 8 (2010), 1362-1376.

Silvano Galliani, Katrin Lasinger, and Konrad Schindler. 2015. Massively Parallel Multiview Stereopsis by Surface Normal Diffusion. The IEEE International Conference on Computer Vision (ICCV) (2015).

Clément Godard, Oisin Mac Aodha, and Gabriel J. Brostow. 2017. Unsupervised Monocular Depth Estimation with Left-Right Consistency. CVPR (2017).

M. Goesele, N. Snavely, B. Curless, H. Hoppe, and S.M. Seitz. 2007. Multi-View Stereo for Community Photo Collections. (2007), 1-8.

Google. 2015. Carboard Camera. https://googleblog.blogspot.com/2015/12/ step-inside-your-photos-with-cardboard.html/. (2015). Accessed: 2016-12-26

Peter Hedman, Tobias Ritschel, George Drettakis, and Gabriel Brostow. 2016. Scalable Inside-out Image-based Rendering. ACM Trans. Graph. 35, 6 (2016), 231:1-231:11.

Sunghoon Im, Hyowon Ha, François Rameau, Hae-Gon Jeon, Gyeongmin Choe, and In So Kweon. 2016. All-Around Depth from Small Motion with a Spherical Panoramic Camera. European Conference on Computer Vision (ECCV '16) (2016), 156-172.

Hiroshi Ishiguro, Masashi Yamamoto, and Saburo Tsuji. 1990. Omni-directional stereo for making global map. In Third International Conference on Computer Vision. IEEE $540-547$.

Shahram Izadi, David Kim, Otmar Hilliges, David Molyneaux, Richard Newcombe, Pushmeet Kohli, Jamie Shotton, Steve Hodges, Dustin Freeman, Andrew Davison and Andrew Fitzgibbon. 2011. KinectFusion: Real-time 3D Reconstruction and Interaction Using a Moving Depth Camera. Proceedings of the 24th Annual ACM Symposium on User Interface Software and Technology (2011), 559-568.

Michal Jancosek and Tomas Pajdla. 2011. Multi-view Reconstruction Preserving Weaklysupported Surfaces. IEEE Conference on Computer Vision and Pattern Recognition (CVPR 2011) (2011), 3121-3128.

Kevin Karsch, Varsha Hedau, David Forsyth, and Derek Hoiem. 2011. Rendering Synthetic Objects into Legacy Photographs. ACM Trans. Graph. 30, 6 (2011), 157:1$157: 12$.

Michael Kazhdan and Hugues Hoppe. 2013. Screened Poisson Surface Reconstruction. ACM Trans. Graph. 32, 3 (2013), article no. 29.

Erum Arif Khan, Erik Reinhard, Roland W. Fleming, and Heinrich H. Bülthoff. 2006. Image-based Material Editing. ACM Transactions on Graphics (Proc. SIGGRAPH 2006) 25,3 (2006), 654-663.

Vladimir Kolmogorov and Ramin Zabih. 2004. What energy functions can be minimized via graph cuts? IEEE Transactions on Pattern Analysis and Machine Intelligence 26, 2 (2004), 65-81

Nikos Komodakis and Georgios Tziritas. 2007. Approximate Labeling via Graph Cuts Based on Linear Programming. IEEE Transactions on Pattern Analysis and Machin Intelligence 29, 8 (2007), 1436-1453.

Johannes Kopf, Michael F. Cohen, Dani Lischinski, and Matt Uyttendaele. 2007. Joint Bilateral Upsampling. ACM Trans. Graph. 26, 3 (2007).

Johannes Kopf, Fabian Langguth, Daniel Scharstein, Richard Szeliski, and Michael Goesele. 2013. Image-based Rendering in the Gradient Domain. ACM Trans. Graph. 32, 6 (2013), 199:1-199:9.

Vivek Kwatra, Arno Schödl, Irfan Essa, Greg Turk, and Aaron Bobick. 2003. Graphcut Textures: Image and Video Synthesis Using Graph Cuts. ACM Trans. Graph. 22, 3 (2003), 277-286.

Fabian Langguth, Kalyan Sunkavalli, Sunil Hadap, and Michael Goesele. 2016. Shadingaware Multi-view Stereo. Proceedings of the European Conference on Computer Vision $(E C C V)(2016)$

Anat Levin, Dani Lischinski, and Yair Weiss. 2004. Colorization Using Optimization. ACM Trans. Graph. 23, 3 (2004), 689-694.

Kaimo Lin, Nianjuan Jiang, Loong-Fah Cheong, Minh N. Do, and Jiangbo Lu. 2016. SEAGULL: Seam-Guided Local Alignment for Parallax-Tolerant Image Stitching 14th European Conference on Computer Vision (ECCV) (2016), 370-385.

Sheng-Jie Luo, I-Chao Shen, Bing-Yu Chen, Wen-Huang Cheng, and Yung-Yu Chuang. 2012. Perspective-aware Warping for Seamless Stereoscopic Image Cloning. ACM Trans. Graph. 31, 6 (2012), article no. 182

Ziyang Ma, Kaiming He, Yichen Wei, Jian Sun, and Enhua Wu. 2013. Constant Time Weighted Median Filtering for Stereo Matching and Beyond. In IEEE International Conference on Computer Vision (ICCV 2013). 49-56.

Raúl Mur-Artal and Juan D. Tardós. 2016. ORB-SLAM2: an Open-Source SLAM System for Monocular, Stereo and RGB-D Cameras. arXiv preprint arXiv:1610.06475 (2016).
OpenMVS. 2016. OpenMVS: open Multi-View Stereo reconstruction library. https: //github.com/cdcseacave/openMVS. (2016). Accessed: 2016-12-26.

Shmuel Peleg and Moshe Ben-Ezra. 1999. Stereo panorama with a single camera. IEEE Conference on Computer Vision and Pattern Recognition (CVPR 1999) (1999), 395-401.

Shmuel Peleg, Moshe Ben-Ezra, and Yael Pritch. 2001. Omnistereo: panoramic stereo imaging. IEEE Transactions on Pattern Analysis and Machine Intelligence 23, 3 (2001), $279-290$.

Realities. 2017. realities.io | Go Places. http://realities.io/. (2017). Accessed: 2017-1-12

Christoph Rhemann, Asmaa Hosni, Michael Bleyer, Carsten Rother, and Margit Gelautz. 2011. Fast cost-volume filtering for visual correspondence and beyond. In IEEE Conference on Computer Vision and Pattern Recognition (CVPR 2011). 3017-3024.

Christian Richardt, Yael Pritch, Henning Zimmer, and Alexander Sorkine-Hornung. 2013. Megastereo: Constructing High-Resolution Stereo Panoramas. IEEE Conference on Computer Vision and Pattern Recognition (CVPR 2013) (2013), 1256-1263.

Daniel Scharstein and Richard Szeliski. 2002. A Taxonomy and Evaluation of Dense Two-Frame Stereo Correspondence Algorithms. International fournal of Computer Vision 47, 1-3 (2002), 7-42.

Frank Schmitt and Lutz Priese. 2009. Sky detection in CSC-segmented color images. International Conference on Computer Vision Theory and Applications (VISAPP 2009) (2009), 101-106.

Johannes Lutz Schönberger, Enliang Zheng, Marc Pollefeys, and Jan-Michael Frahm. 2016. Pixelwise View Selection for Unstructured Multi-View Stereo. European Conference on Computer Vision (ECCV) (2016).

Steven M Seitz, Brian Curless, James Diebel, Daniel Scharstein, and Richard Szeliski. 2006. A comparison and evaluation of multi-view stereo reconstruction algorithms. In 2006 IEEE Computer Society Conference on Computer Vision and Pattern Recognition (CVPR'06), Vol. 1. IEEE, 519-528.

Jonathan Shade, Steven Gortler, Li-wei He, and Richard Szeliski. 1998. Layered Depth Images. Proceedings of SIGGRAPH '98 (1998), 231-242.

Harry Shum and Rick Szeliski. 1998. Construction and refinement of panoramic mosaics with global and local alignment. Sixth International Conference on Computer Vision (ICCV'98) (1998), 953-958

Richard Szeliski. 2006. Image Alignment and Stitching: A Tutorial. Found. Trends. Comput. Graph. Vis. 2, 1 (2006), 1-104.

Jayant Thatte, Jean-Baptiste Boin, Haricharan Lakshman, and Bernd Girod. 2016. Depth augmented stereo panorama for cinematic virtual reality with head-motion parallax. 2016 IEEE International Conference on Multimedia and Expo (ICME) (2016).

Benjamin Ummenhofer and Thomas Brox. 2015. Global, Dense Multiscale Reconstruction for a Billion Points. IEEE International Conference on Computer Vision (ICCV) (2015)

Benjamin Ummenhofer, Huizhong Zhou, Jonas Uhrig, Nikolaus Mayer, Eddy Ilg, Alexey Dosovitskiy, and Thomas Brox. 2017. DeMoN: Depth and Motion Network for Learning Monocular Stereo. IEEE Conference on Computer Vision and Pattern Recognition (CVPR) (2017).

Valve. 2016. Valve Developer Community: Advanced Outdoors Photogrammetry. https://developer.valvesoftware.com/wiki/Destinations/Advanced_Outdoors_ Photogrammetry. (2016). Accessed: 2016-11-3.

George Vogiatzis, Carlos Hernández Esteban, Philip H. S. Torr, and Roberto Cipolla. 2007. Multiview Stereo via Volumetric Graph-Cuts and Occlusion Robust PhotoConsistency. IEEE Trans. Pattern Anal. Mach. Intell. 29, 12 (2007), 2241-2246.

Michael Waechter, Mate Beljan, Simon Fuhrmann, Nils Moehrle, Johannes Kopf, and Michael Goesele. 2017. Virtual Rephotography: Novel View Prediction Error for 3D Reconstruction. ACM Trans. Graph. 36, 1 (2017), article no. 8.

Michael Waechter, Nils Moehrle, and Michael Goesele. 2014. Let There Be Color! Large-Scale Texturing of 3D Reconstructions. ECCV 20148693 (2014), 836-850.

Katja Wolff, Changil Kim, Henning Zimmer, Christopher Schroers, Mario Botsch, Olga Sorkine-Hornung, and Alexander Sorkine-Hornung. 2016. Point Cloud Noise and Outlier Removal for Image-Based 3D Reconstruction. In International Conference on 3 D Vision (3DV 2016). 118-127.

Chenglei Wu, Bennet Wilburn, Yasuyuki Matsushita, and Christian Theobalt. 2011. High-quality Shape from Multi-view Stereo and Shading Under General Illumination. IEEE Conference on Computer Vision and Pattern Recognition (CVPR '11) (2011), 969976.

Kuk-Jin Yoon and In-So Kweon. 2005. Locally adaptive support-weight approach for visual correspondence search. In IEEE Conference on Computer Vision and Pattern Recognition (CVPR 2005), Vol. 2. 924-931.

Julio Zaragoza, Tat-Jun Chin, Michael S. Brown, and David Suter. 2013. As-Projective-AsPossible Image Stitching with Moving DLT. Proceedings of the 2013 IEEE Conference on Computer Vision and Pattern Recognition (2013), 2339-2346.

Fan Zhang and Feng Liu. 2014. Parallax-Tolerant Image Stitching. Proceedings of the 2014 IEEE Conference on Computer Vision and Pattern Recognition (2014), 3262-3269.

Fan Zhang and Feng Liu. 2015. Casual Stereoscopic Panorama Stitching. IEEE Conference on Computer Vision and Pattern Recognition (CVPR '15) (2015), 2002-2010.

Ke Colin Zheng, Sing Bing Kang, Michael F. Cohen, and Richard Szeliski. 2007. Layered Depth Panoramas. IEEE Conference on Computer Vision and Pattern Recognition (CVPR 2007) (2007), 1-8.

C. Lawrence Zitnick, Sing Bing Kang, Matthew Uyttendaele, Simon Winder, and Richard Szeliski. 2004. High-quality Video View Interpolation Using a Layered Representation. ACM Trans. Graph. (Proc. SIGGRAPH 2004) 23, 3 (2004), 600-608. 


\section{A PLANE-SWEEP MVS IMPLEMENTATION}

Depth Hypotheses: We distribute $N=220$ depth hypotheses between $d_{\text {min }}=0.5 \mathrm{~m}$ and $d_{\max }=1500 \mathrm{~m}$ according to the square root of their disparities $\left(\propto \sqrt{d^{-1}}\right)$, striking a balance between uniform depth intervals $(\propto d)$ which undersamples foreground depths and uniform disparity intervals $\left(\propto d^{-1}\right)$ which in turn oversamples nearby regions with sub-centimeter accuracy.

Smoothness Term $E_{\text {smooth }}(i, j)$ : Our smoothness energy

$$
E_{\text {smooth }}(i, j)=w_{\text {color }}\left(c_{i}, c_{j}\right) w_{\text {depth }}\left(d_{i}, d_{j}\right)
$$

encourages the resulting depth map to be smooth wherever the image lacks texture. It is a weighted sum over all neighboring pixels $(i, j)$, where

$$
w_{\text {color }}\left(c_{i}, c_{j}\right)=\alpha_{c}+\left(1-\alpha_{c}\right) e^{\frac{-\left|c_{i}-c_{j}\right|^{2}}{2 \sigma_{c}^{2}}}
$$

down-weighs the smoothness at strong image gradients, and

$$
w_{\text {depth }}=\frac{\left|d_{i}-d_{j}\right|+\alpha_{d} \min \left(\left|d_{i}-d_{j}\right|, \tau_{d}\right)}{Z}
$$

is a piece-wise linear smoothness energy on depth labels, which grows faster near zero. In all experiments, we set $\alpha_{c}=0.05, \sigma_{c}=$ $0.033, \tau_{d}=16$ and $\alpha_{c}=31$. We normalize $w_{\text {depth }}$ to be between 0 and 1 using $Z=N\left(\alpha d \tau_{d}+1\right)=715$.

Photo-consistency Term $E_{\text {photo }}(i)$ : To evaluate photoconsistency, we project a pixel $i$ into 16 neighboring images, where we compare both colors and image gradients using sum-of-absolute-differences (SAD). We use the transformation $f(x)=1-\exp \left(-x / \sigma_{\text {photo }}\right)$ to truncate both SAD costs and measure the photoconsistency against one of the neighbor images $n$ as the weighted average $\alpha_{\nabla} f\left(S A D_{\nabla}^{n}\right)+\left(1-\alpha_{\nabla}\right) f\left(S A D_{c}^{n}\right)$. We then compute the overall photoconsistency of the depth assignment $d(i)$ by averaging the $50 \%$ lowest photoconsistency energies from all neighbor images. In all experiments, we set $\alpha_{\nabla}=0.9$ and $\sigma_{\text {photo }}=0.033$.

Finally, we filter each slice of the resulting cost volume using

(1) A $5 \times 5$ Shiftable matching window [Bobick and Intille 1999], and

(2) Cost volume filtering [Rhemann et al. 2011; Yoon and Kweon 2005] with a 10px standard deviation and $\sigma_{c}=0.033$.

In general, cost-volume filtering enables us to smooth the cost while preserving important objects edges. However, in our experiments we found that this filter is sensitive to large color variations and can make the filter footprint very small for small patches of unusual color. This is why we apply shiftable-matching windows, essentially prefiltering the cost volume across such small patches.

Photo-consistency Confidence $w_{\text {photo }}(i)$ : Photo-consistency measures are less reliable when a pixel is visible in a small number of views: With more views, repeating patterns are no longer ambiguous. Textureless surfaces also become less ambiguous, as the textured parts in each view rule out locations where such surfaces cannot be.

To avoid mistakes for pixels visible in few views, we limit the influence of the photo-consistency term for such pixels, and rely more on the smoothness term to resolve their depths. Concretely, for each pixel $i$, we try all $N$ depth hypotheses, and record $M_{i}$, the maximum number of views that could see it. We then compute the confidence

$$
w_{\text {photo }}(i)=w_{\min }+\left(1-w_{\min }\right) \operatorname{clamp}_{[0,1]}\left(\frac{M_{i}-M_{\min }}{M_{\max }-M_{\min }}\right),
$$

a linear ramp between 1 for reliable pixels seen in $M_{\max }=8$ or more images, to a minimum confidence $w_{\text {min }}=0.1$ for pixels seen in $M_{\text {min }}=4$ images or fewer.

Geometric Consistency Filter: We use geometric consistency (GC) filters [Wolff et al. 2016] for two components of our algorithm: identifying reliable anchor pixels when computing the near envelope (Section 4.3.2) and down-weighting unreliable labels during front and back layer stitching (Section 4.4.2).

The general idea behind GC filters is to discard pixels whose depths do not agree between different depth maps. In our implementation, we project each pixel into 16 neighboring depth maps and check whether it incorrectly occludes another view. We determine whether a pixel $i$ agrees with another depth map $d^{\prime}$ with the ratio

$$
r=\frac{d^{\prime}(p(i))}{p(i) \cdot z}
$$

between the depth $d^{\prime}(p(i))$ stored in the depth map and depth of $i$ as seen in $d^{\prime}$. If $r>0.75$, we assume $i$ to be in front of the surfaces in $d^{\prime}$, Similarly, if $r<0.75^{-1}$, then $i$ is behind.

Based on this, we label pixels as invalid using simple thresholds:

- If it lands between neighboring camera and estimated surface in at least $G C_{\text {conflicts }}$ images, mark the pixel as invalid.

- If it does not land close (i.e. neither behind or in front) to the surface in at least $G C_{\text {agrees }}$ of the neighboring depth maps, mark it as invalid. The intuition is that stereo matching can only succeed if at least two cameras agree on the depth.

As a final clean-up step, we filter the result to smooth out salt-and pepper noise. For this, we use a weighted median filter [Ma et al. 2013], guided by the depth labels $\left(\sigma_{l}=10\right)$.

During stitching, we use the thresholds

$$
G C_{\text {conflicts }}=3 \text { and } G C_{\text {agrees }}=1 .
$$

For the near envelope, we use more aggressive thresholds

$$
G C_{\text {conflicts }}=1 \text { and } G C_{\text {agrees }}=2,
$$

when computing reliable anchor points. 